\title{
A set of moves for Johansson representation of 3-manifolds
}

\author{
by \\ Rubén Vigara (Madrid)
}

\begin{abstract}
A Dehn sphere $\Sigma$ in a closed 3 -manifold $M$ is a 2 -sphere immersed in $M$ with only double curve and triple point singularities. The Dehn sphere $\Sigma$ fills $M$ if it defines a cell decomposition of $M$. The inverse image in $S^{2}$ of the double curves of $\Sigma$ is the Johansson diagram of $\Sigma$ and if $\Sigma$ fills $M$ it is possible to reconstruct $M$ from the diagram. A Johansson representation of $M$ is the Johansson diagram of a filling Dehn sphere of $M$. Montesinos proved that every closed 3-manifold has a Johansson representation coming from a nullhomotopic filling Dehn sphere. In this paper a set of moves for Johansson representations of 3-manifolds is given. This set of moves suffices for relating different Johansson representations of the same 3-manifold coming from nullhomotopic filling Dehn spheres. The proof of this result is outlined here.
\end{abstract}

1. Introduction. Throughout the paper all 3-manifolds are assumed to be closed, that is, compact, connected and without boundary, and all surfaces are assumed to be compact and without boundary. A surface may have more than one connected component. We will denote a 3-manifold by $M$ and a surface by $S$.

Let $M$ be a 3 -manifold.

A subset $\Sigma \subset M$ is a Dehn surface in $M$ (see $[\mathrm{P}]$ ) if there exists a surface $S$ and a transverse immersion $f: S \rightarrow M$ such that $\Sigma=f(S)$. In this situation we say that $f$ parametrizes $\Sigma$. If $S$ is a 2 -sphere then $\Sigma$ is a Dehn sphere. For a Dehn surface $\Sigma \subset M$, its singularities are divided into double points (Figure 1(a)), and triple points (Figure 1(b)), and they are arranged along double curves (see Section 2 below for definitions). A Dehn surface $\Sigma \subset M$ fills $M$ [Mo2] if it defines a cell decomposition of $M$ in which the 0 -skeleton is the set of triple points of $\Sigma$; the 1-skeleton is the set of double and triple points of $\Sigma$; and the 2-skeleton is $\Sigma$ itself. Filling Dehn

2000 Mathematics Subject Classification: 57N10, 57N35, 57M99.

Key words and phrases: representation of 3-manifolds, set of moves, immersed surfaces, Dehn surfaces, Johansson diagram, double curves, triple points.

This research has been partially supported by a predoctoral grant from the U.N.E.D. (1999). 


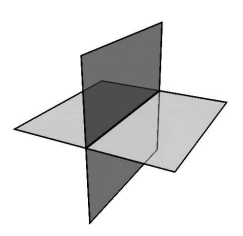

(a)

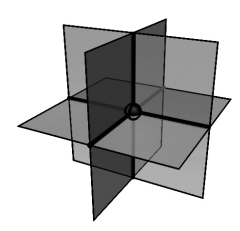

(b)

Fig. 1

spheres of 3-manifolds are defined in [Mo2] following ideas of W. Haken (see $[\mathrm{Ha}])$. In $[\mathrm{F}-\mathrm{R}]$ it is proved that every closed orientable 3 -manifold has a Dehn sphere whose complement is a union of open 3-balls. In [Mo2] the following theorem is proved (see also [V1]):

THEOREM 1 ([Mo2]). Every closed orientable 3-manifold has a nullhomotopic filling Dehn sphere.

A filling Dehn sphere is nullhomotopic if one (and hence any) of its parametrizations is nullhomotopic, that is, homotopic to a constant map.

Let $\Sigma \subset M$ be a filling Dehn sphere and $f: S^{2} \rightarrow M$ a transverse immersion parametrizing $\Sigma$. In this case we say that $f$ is a filling immersion. The inverse image by $f$ in $S^{2}$ of the set of double and triple points of $\Sigma$ is the singular set of $f$. The singular set of $f$, together with the information on how its points are identified by $f$ in $M$, is the Johansson diagram of $\Sigma$ in the terminology of [Mo2]. As stated in [Mo2], for a given diagram in $S^{2}$ it is possible to find if it is the Johansson diagram for a filling Dehn sphere $\Sigma$ in some 3-manifold $M$. If this is the case, it is also possible to reconstruct such an $M$ from the diagram. Thus, Johansson diagrams are a suitable way for representing all closed, orientable 3-manifolds and it is interesting to further study them. For a 3-manifold $M$, we say that a Johansson diagram of a filling Dehn sphere of $M$ is a Johansson representation of $M$ (see [Mo2]). In [Mo2] an algorithm is given for obtaining a Johansson representation of a closed orientable 3-manifold $M$ from any Heegaard diagram of $M$. A simpler algorithm is given in [V1]. In both papers, the Johansson representations obtained come from nullhomotopic filling Dehn spheres of $M$.

We will deal here with the problem of deciding how different Johansson representations of the same 3-manifold are related to each other. With this problem in mind, we study how different filling Dehn spheres of the same 3-manifold are related to each other. In [V2], the following theorem is proved.

Theorem 2. Let $M$ be a closed 3-manifold. Let $f, g: S^{2} \rightarrow M$ be two nullhomotopic filling immersions. Then there is a finite sequence of filling immersions $f=f_{0}, f_{1}, \ldots, f_{n}=g$ such that for each $i=0, \ldots, n-1$ the 
immersions $f_{i}$ and $f_{i+1}$ differ by an ambient isotopy of $S^{2}$, or by an ambient isotopy of $M$, or by one of the moves depicted in Figure 2.

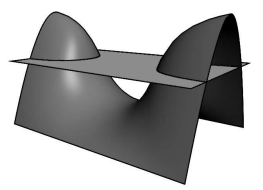

(a)

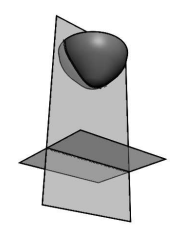

(b)

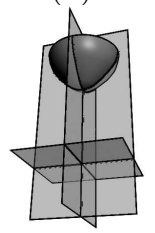

(c)
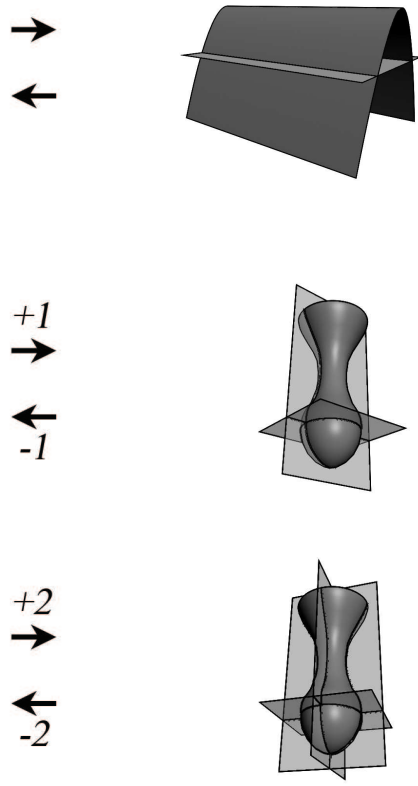

Fig. 2

This theorem gives a complete set of moves for relating Johansson representations of the same 3-manifold coming from nullhomotopic filling Dehn spheres (see Corollary 39).

The detailed proof of Theorem 2 is quite long, and it uses both smooth and combinatorial techniques. In this paper we will give an outline of this proof. The paper is organized as follows.

In Section 2, we give some preliminary definitions about Dehn surfaces and cell complexes. Most of Section 3 and Sections 4 to 10 introduce some partial results needed to sketch the proof of Theorem 2. This sketch is given in Section 11. A reader wishing to skip the details can jump directly from Section 3 to Section 12.

The proof of Theorem 2 in [V2] relies on three Key Lemmas that we will state here without proof. In Section 3 we present some results about regular homotopies of immersions of surfaces in 3-manifolds, and we introduce the concept of filling-preserving moves and filling homotopy for filling immersions. Key Lemma 1 is stated in Section 4, where we define the modifications of immersions of surfaces in 3-manifolds by pushing disks. This 
kind of modification was defined in [H-N1], and Key Lemma 1 asserts that certain regular homotopies can be decomposed into pushing disks with some nice properties.

In Section 5 we introduce a surgery method for modifying Dehn surfaces that will be useful later, and in Section 6 we present three examples of modifications of filling Dehn surfaces that can be done using only the fillingpreserving moves defined in Section 3.

In Section 7 we introduce some combinatorial tools that will be essential in Key Lemmas 2 and 3: the concept of shelling of a cell complex and the concept of simplicial collapsing for a simplicial complex. These concepts will appear almost everywhere in Sections 8 to 11. In the same Section 7 we introduce smooth triangulations of manifolds, which gives us a theoretical basis for applying the previously defined combinatorial concepts to our case.

We explain in Section 8 how any smooth triangulation $T$ of a 3 -manifold $M$ can be "inflated" to obtain a filling Dehn sphere of $M$. This inflated sphere is transverse to any Dehn sphere of $M$ that lies in the 2-skeleton of $T$. When the triangulation $T$ of $M$ is "sufficiently good" with respect to a filling Dehn surface $\Sigma$ of $M$ we can use it to obtain from $\Sigma$ other filling surfaces "as complicated as we want" using only filling-preserving moves. These constructions are used in Key Lemma 2, which is also stated in Section 8. Every pushing disk transformation (as defined in Section 4) of a Dehn surface can be performed by regular homotopy. For a given pushing disk transformation, Key Lemma 2 proposes a new scenario in which the pushing disk transformation can be performed by filling homotopy.

In Section 9 we discuss briefly how two filling Dehn spheres of the same 3-manifold can intersect each other, and this discussion is used in Section 10, where we state Key Lemma 3. It ensures that when two filling Dehn surfaces intersect in a "sufficiently good" way, the inflating constructions introduced in Section 8 can be made simultaneously for one of them and for the union of both.

All the constructions that we have qualified above as "sufficiently good" are intimately related to the concept of shelling.

In Section 12 we translate Theorem 2 into Johansson representations of 3-manifolds and we give some examples, and in Section 13 we explain briefly how we can obtain a nullhomotopic Johansson representation of a 3-manifold $M$ from any Johansson representation of $M$.

In the final Section 14 we give a brief discussion of some related problems.

This paper is part of the Ph.D. thesis [V2] of the author, which has been written under the supervision of Prof. J. M. Montesinos. I am very grateful to him for all his valuable advice, specially for his suggestions and comments during the writing of this paper and his careful reading of the 
previous versions of this manuscript. I would also like to thank the referee for his suggestions and the editor for pointing out some misprints in the final version of this paper.

2. Preliminaries. Because our starting point is Theorem 4 below, we will work in the smooth category. Nevertheless, if one could check that the analogue of Theorem 4 in the PL category is true (we do not know of any reference), all our constructions have their translation to the PL case and so Theorem 2 would also be true in the PL case.

Thus, all the manifolds are assumed to be equipped with a smooth structure and maps between two manifolds are assumed to be smooth.

For the standard definitions of differential topology (immersions, transversality, etc.), see [Hi] or [G-P], for example. For a general treatment of PL topology we refer to $[\mathrm{Hu}]$, for example.

For a subset $X$ of a manifold, we denote the interior, closure and boundary of $X$ by $\operatorname{int}(X), \operatorname{cl}(X)$ and $\partial X$ respectively.

Let $A$ and $B$ be two sets. For a map $f: A \rightarrow B$ the singular values or singularities of $f$ are the points $x \in B$ with $\#\left\{f^{-1}(x)\right\}>1$, and the singular points of $f$ are the inverse image points under $f$ of the singularities of $f$. The singular set $S(f)$ of $f$ is the set of singular points of $f$ in $A$, and the singularity set $\bar{S}(f)$ of $f$ is the set of singularities of $f$ in $B$. Of course $f(S(f))=\bar{S}(f)$. This notation is similar to but slightly different from that of $[\mathrm{Sh}]$.

From now on, $M$ will denote a 3 -manifold as at the beginning of Section 1.

Let $\Sigma$ be a Dehn surface in $M$. Let $S$ be a surface and $f: S \rightarrow M$ a transverse immersion parametrizing $\Sigma$. In this case we say that $S$ is the domain of $\Sigma$. For any $x \in M$ we have $\#\left\{f^{-1}(x)\right\} \leq 3$ (see [He]). The singularities of $f$ are divided into double points of $f$, with $\#\left\{f^{-1}(x)\right\}=2$, and triple points of $f$, with $\#\left\{f^{-1}(x)\right\}=3$. A small neighbourhood of a double or a triple point looks as in Figures 1(a) and 1(b) respectively. The singularity set $\bar{S}(f)$ of $f$, the set of triple points of $f$, and the domain $S$ (up to homeomorphism) do not depend upon the parametrization $f$ of $\Sigma$. We define the singularity set of $\Sigma$, denoted by $\bar{S}(\Sigma)$, to be the singularity set of any parametrization of $\Sigma$. A double curve of $\Sigma$ is the image of an immersion $\bar{\gamma}: S^{1} \rightarrow M$ contained in the singularity set of $\Sigma$ (see [Sh]). The singularity set of $\Sigma$ is the union of the double curves of $\Sigma$. Because $S$ is compact, $\Sigma$ has a finite number of double curves. Following [Sh], we denote by $T(\Sigma)$ the set of triple points of $\Sigma$. The Dehn surface $\Sigma$ is embedded if its singularity set is empty. A standardly embedded 2-sphere in $M$ is a 2 -sphere embedded in $M$ that bounds a 3-ball in $M$. 
A component of $\Sigma$ is the image under $f$ of a connected component of the domain $S$. Note that the components of $\Sigma$ may not coincide with the connected components of $\Sigma$.

A Dehn surface $\Sigma$ in $M$ fills $M$ if it defines a cell decomposition of $M$ as indicated in Section 1. This definition generalizes to general surfaces a definition given in [Mo2] for Dehn spheres.

The following trivial proposition gives an equivalent definition of filling Dehn surface.

Proposition 3. $\Sigma$ fills $M$ if and only if

(1) $M-\Sigma$ is a disjoint union of open 3-balls,

(2) $\Sigma-\bar{S}(\Sigma)$ is a disjoint union of open 2-disks,

(3) $\bar{S}(\Sigma)-T(\Sigma)$ is a disjoint union of open intervals.

The following statements and definitions for cell complexes are also valid for simplicial complexes. We consider the cells of a cell complex as open cells. If $K$ is a cell complex, and $\varepsilon, \varepsilon^{\prime}$ are two cells of $K$, we write $\varepsilon<\varepsilon^{\prime}$ when $\varepsilon$ is a face of $\varepsilon^{\prime}$, that is, when $\operatorname{cl}(\varepsilon) \subset \operatorname{cl}\left(\varepsilon^{\prime}\right)$. The cells $\varepsilon$ and $\varepsilon^{\prime}$ are incident if $\varepsilon<\varepsilon^{\prime}$ or $\varepsilon^{\prime}<\varepsilon$, and adjacent if $\operatorname{cl}(\varepsilon) \cap \operatorname{cl}\left(\varepsilon^{\prime}\right) \neq \emptyset$. For a cell $\varepsilon$ of $K$, we define the (open) star of $\varepsilon$ as the union of all cells $\varepsilon^{\prime}$ of $K$ with $\varepsilon<\varepsilon^{\prime}$. The star of $\varepsilon$ is denoted by $\operatorname{star}(\varepsilon)$.

If $\varepsilon$ is a cell of the cell complex $K$, and $P$ is a vertex (0-cell) of $\varepsilon$, we say that $\varepsilon$ is self-adjacent at $P$ if a regular neighbourhood of $P$ in $K$ intersects $\varepsilon$ in more than one connected component. Otherwise we say that $\varepsilon$ is regular at $P$. We say that $\varepsilon$ is regular if it is regular at every vertex of $\varepsilon$. The complex $K$ is regular at $P$ if every cell of $K$ incident with $P$ is regular at $P$, and $K$ is regular if every cell of $K$ is regular (cf. [Ma]). A filling Dehn surface $\Sigma$ of $M$ is regular (regular at a triple point) if the cell decomposition of $M$ that defines $\Sigma$ is regular (at this triple point).

If $\Sigma$ is a filling Dehn surface, then a connected component of $M-\Sigma$ is called a region of $M-\Sigma$, and a connected component of $\Sigma-\bar{S}(\Sigma)$ is sometimes called a face of $\Sigma$.

3. Filling homotopy. An ambient isotopy of a manifold $N$ is a map $\varsigma: N \times[0,1] \rightarrow N$ such that $\varsigma_{t}=\varsigma(\cdot, t)$ is a diffeomorphism for each $t \in[0,1]$ and $\varsigma_{0}=\operatorname{id}_{N}$. Two immersions $f, g: S \rightarrow M$ are ambient isotopic in $M$ if there is an ambient isotopy $\bar{\varsigma}$ of $M$ with $\bar{\varsigma}_{1} \circ f=g$. The same immersions are ambient isotopic in $S$ if there is an ambient isotopy $\varsigma$ of $S$ with $f \circ \varsigma_{1}=g$. We generally say that $f$ and $g$ are ambient isotopic if they are related by ambient isotopies of $S$ and ambient isotopies of $M$.

Two immersions $f, g: S \rightarrow M$ from a surface $S$ into the 3 -manifold $M$ are regularly homotopic if there is a homotopy $H: S \times[0,1] \rightarrow M$ with $H(\cdot, 0)=f$ and $H(\cdot, 1)=g$ such that $H(\cdot, t)$ is an immersion for each 
$t \in[0,1]$. The homotopy $H$ defines a smooth path of immersions from $S$ into $M$ having $f$ and $g$ as its endpoints. If $f$ and $g$ are regularly homotopic, they are indeed homotopic. The converse is not true in general. Nevertheless, an immediate corollary of Theorem 1.1 in $[\mathrm{H}-\mathrm{H}]$ or Theorem 6 in $[\mathrm{L}]$ is:

THEOREM 4. Two immersions from $S^{2}$ into a 3-manifold are regularly homotopic if and only if they are homotopic.

In particular, two parametrizations of nullhomotopic filling Dehn spheres of $M$ must be regularly homotopic.

In [H-N1] a set of elementary deformations for immersions of surfaces in 3-manifolds is introduced. This set of moves is composed by the saddle move (called an elementary deformation of type VI in [H-N1]) of Figure 2(a), together with the moves depicted in Figure 3. We will call these elementary deformations the Homma-Nagase moves. In [H-N2] the following is proved:

THEOREM 5. Two transverse immersions from a closed surface $S$ into a 3-manifold $M$ are regularly homotopic if and only if we can deform them into each other by a finite sequence of Homma-Nagase moves, together with ambient isotopies of $M$.

The proof of this theorem in [H-N2] is in the PL category. A proof of the smooth version is indicated in $[\mathrm{R}]$. An equivalent result, also in the differentiable case, is Theorem 3.1 of $[\mathrm{H}-\mathrm{H}]$.

We will propose another set of moves (Haken moves), which is the result of substituting in the Homma-Nagase set of moves the moves of Figures $3(\mathrm{~b})$ and 3(c) by the finger moves 1 and 2 depicted in Figures 2(b) and 2(c) respectively. The following lemma can be easily proved:

Lemma 6. The Homma-Nagase set of moves and the Haken set of moves are equivalent.

To prove this lemma it must be shown that each Homma-Nagase move can be obtained using Haken moves (and ambient isotopies) and vice versa. Thus, in Theorem 5 we can substitute the Homma-Nagase moves by the Haken moves.

The Haken moves are more suitable than the Homma-Nagase moves when dealing with filling Dehn surfaces. In the Haken set of moves, the move of Figure 3(a) is called a finger move 0 . For $i=0,1,2$ a finger move $i$ is a finger move $+i$ when it happens from left to right in the figure, and a finger move $-i$ if it happens in the opposite sense. A saddle move is equivalent (symmetric) in both senses.

Lemma 7. Let $f, g: S \rightarrow M$ be two immersions. Then:

(1) if $f$ and $g$ are related by a finger move 0 , then one of them is not a filling immersion; 


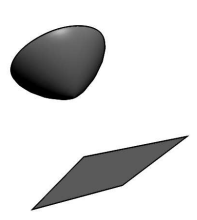

(a)

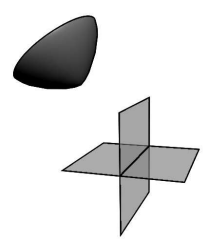

(b)

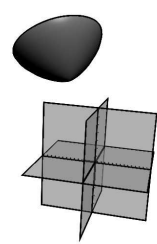

(c)
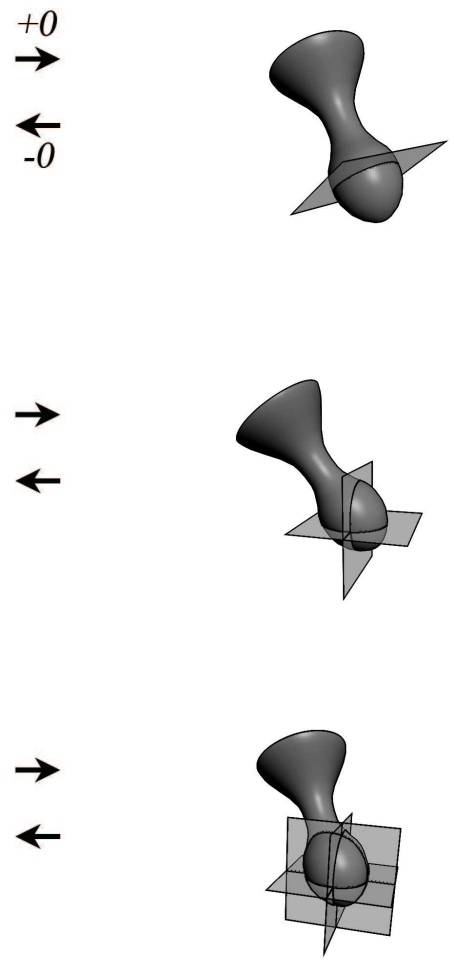

Fig. 3

(2) if $g$ is obtained from $f$ by applying a finger move +1 or \pm 2 and $f$ is a filling immersion, then $g$ is a filling immersion;

(3) if $g$ is obtained from $f$ by applying a finger move -1 or a saddle move and $f$ is a filling immersion, then $g$ is not necessarily a filling immersion.

This lemma can be proved by inspection, using the characterization of filling immersions given by Proposition 3 .

Lemma 7 inspired the following definition. If $f: S \rightarrow M$ is a filling immersion and we modify $f$ by a Haken move, we say that the move is fillingpreserving if the immersion $g$ we get after the move is again a filling immersion. In this terminology, Lemma 7 states that a finger move 0 cannot be filling-preserving; that finger moves +1 and \pm 2 are always filling-preserving; and that finger moves -1 and saddle moves are sometimes filling-preserving and sometimes not. The next step is the following definition:

Definition 8. Let $f, g: S \rightarrow M$ be two filling immersions. We say that $f$ and $g$ are filling homotopic if there is a finite sequence $f=f_{0}, f_{1}, \ldots, f_{n}=g$ 
of immersions such that for each $i=0, \ldots, n-1$ the immersions $f_{i}$ and $f_{i+1}$ are ambient isotopic or related by a filling-preserving move.

Note that in the above definition, all $f_{0}, \ldots, f_{n}$ are filling immersions. In these terms, Theorem 2 can be restated as follows:

ThEOREM 9. If $f, g: S^{2} \rightarrow M$ are nullhomotopic filling immersions, then they are filling homotopic.

This gives a partial answer to the following conjecture:

CONJECTURE 10. Regularly homotopic filling immersions of arbitrary surfaces are filling homotopic.

The proof of Theorem 2 given in [V2] and sketched here can perhaps be adapted to a more general case but we still do not know how to do this.

4. Pushing disks. Let $f, g: S \rightarrow M$ be two immersions. Assume that there is a closed disk $D \subset S$ such that:

(1) $f$ and $g$ agree in $S-D$;

(2) $\left.f\right|_{D}$ and $\left.g\right|_{D}$ are both embeddings;

(3) $f(D)$ and $g(D)$ intersect only in $f(\partial D)=g(\partial D)$;

(4) $f(D) \cup g(D)$ bounds a 3-ball $B$ in $M$ (Figure 4).

Then we say that $g$ is obtained from $f$ by pushing the disk $D$ through $B$ or along $B$ (see Figure 4 ). The pair $(D, B)$ is a pushing disk (see [H-N1]). In the pushing disk $(D, B)$, the disk $D$ is the pushed disk, $B$ is the pushing ball and we also say that $f(\partial D)=g(\partial D)$ is the equator of $B$, denoted by eq $(B)$. If both $f$ and $g$ are transverse immersions, we say that the pushing disk $(D, B)$ is transverse. In the pushing disk $(D, B)$, the "rest" of the immersed surface, $f(S-D)$, may intersect $B$ in any manner (Figure 4(b)). If we are given the immersion $f$ and the pushing disk $(D, B)$, then the immersion $g$ is well defined up to an ambient isotopy of $S$.

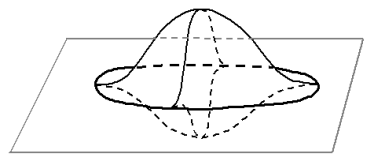

(a)

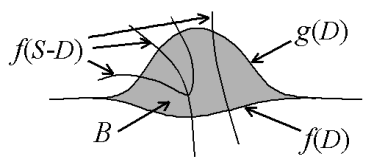

(b)

Fig. 4

We will say that two (transverse) immersions $f, g: S \rightarrow M$ are regularly homotopic by (transverse) pushing disks if there is a finite sequence $f=$ $f_{0}, f_{1}, \ldots, f_{n}=g$ of (transverse) immersions such that $f_{i}$ is obtained from $f_{i-1}$ by a pushing disk for $i=1, \ldots, n$. 
The first step in the proof of Theorem 2 is the following lemma, whose proof is in [V2].

Lemma 11 (Key Lemma 1). Let $f, g: S^{2} \rightarrow M$ be two nullhomotopic immersions such that $f$ is transverse and $g\left(S^{2}\right)$ is a standardly embedded 2-sphere in $M$. Assume that there exists a closed disk $D \subset S^{2}$ such that $f$ and $g$ agree over $D$. Then $f$ and $g$ are regularly homotopic by transverse pushing disks keeping $D$ fixed.

Note that the Homma-Nagase moves and the Haken moves are special kinds of transverse pushing disks. However, Theorem 5 decomposes a regular homotopy into transverse pushing disks and ambient isotopies of $M$. Disposing of this ambient isotopy is the hardest part in the proof of Key Lemma 1 in [V2]. Just as an immersion behaves locally as an embedding, a regular homotopy behaves locally as an isotopy. Using this, the proof of Key Lemma 1 will be obtained after a detailed study of isotopies of embedded surfaces in 3-manifolds, and it is mainly inspired by [H-Z].

5. Spiral piping. In $[\mathrm{Ba}]$ it is explained how to modify Dehn surfaces by surgery, also called piping (see [R-S, p. 67]). We now introduce a special kind of piping that will be useful later. Let $\Sigma$ be a Dehn surface in $M$, and let $P$ be a triple point of $\Sigma$. If $P$ is the triple point depicted in Figure 5(a), consider the surface $\Sigma^{\prime}$ that is exactly identical with $\Sigma$ except in a neighbourhood of $P$ that can be as small as necessary. In this neighbourhood of $P$, the Dehn surface $\Sigma^{\prime}$ looks like Figure 5(b), and we say that $\Sigma^{\prime}$ is obtained from $\Sigma$ by a spiral piping around $P$.

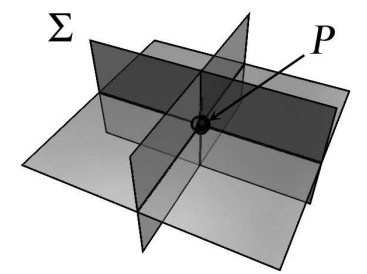

(a)

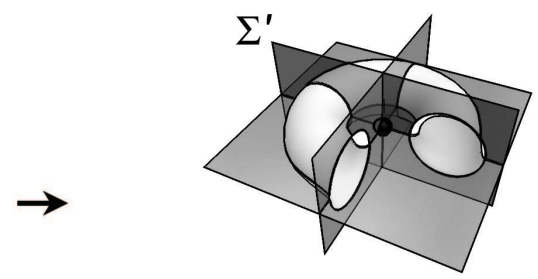

(b)

Fig. 5

Proposition 12. In this situation, if $\Sigma$ is a (regular) filling Dehn surface of $M$, then $\Sigma^{\prime}$ is a (regular) filling Dehn surface of $M$.

See [V2] for more details.

If the two sheets of $\Sigma$ that become connected by the piping (the two vertical sheets in Figure 5) belong to different components $\Sigma_{1}$ and $\Sigma_{2}$ of $\Sigma$, then after performing the spiral piping these two components of $\Sigma$ become a unique component $\Sigma_{1} \# \Sigma_{2}$ of $\Sigma^{\prime}$. 
If $S$ is the domain of $\Sigma$, and $S^{\prime}$ is the domain of $\Sigma^{\prime}$, it is easy to check that $S^{\prime}$ is the result of removing the interior of two small closed disks $\delta_{1}, \delta_{2}$ from $S$ and identifying their boundaries in an appropriate way. If $\delta_{1}$ and $\delta_{2}$ belong to different connected components $S_{1}, S_{2}$ of $S$ respectively, then $S^{\prime}$ is the result of replacing the union $S_{1} \cup S_{2}$ in $S$ by the connected sum $S_{1} \# S_{2}$.

The following definition and theorem appear in [V1].

Definition 13. A Dehn surface $\Sigma \subset M$ that fills $M$ is called a filling collection of spheres in $M$ if its domain is a disjoint union of a finite number of 2-spheres.

THEOREM 14. If $M$ has a filling collection of spheres $\Sigma$, then $M$ has a filling Dehn sphere $\Sigma^{\prime}$. If each component of $\Sigma$ is nullhomotopic, we can choose $\Sigma^{\prime}$ to be nullhomotopic.

Proof. Let $\Sigma$ be a filling collection of spheres in $M$, and let $\Sigma_{1}, \ldots, \Sigma_{m}$ be the different components of $\Sigma$.

The 2-skeleton of any cell decomposition of $M$ is connected because $M$ is connected. Therefore, $\Sigma$ is connected. Thus, we can assume that $\Sigma_{1}, \ldots, \Sigma_{m}$ are ordered in such a way that $\Sigma_{1} \cup \cdots \cup \Sigma_{k}$ is connected for every $k \in$ $\{1, \ldots, m\}$. In particular, $\Sigma_{k}$ intersects $\Sigma_{1} \cup \cdots \cup \Sigma_{k-1}$ for all $k \in\{2, \ldots, m\}$.

Because $\Sigma_{1} \cap \Sigma_{2}$ is nonempty, it contains a double curve of $\Sigma$, and because $\Sigma$ fills $M$, this double curve contains at least one triple point $P$ of $\Sigma$. Connecting $\Sigma_{1}$ and $\Sigma_{2}$ near $P$ by a spiral piping, we obtain a new Dehn sphere $\Sigma_{1} \# \Sigma_{2}$ such that $\left(\Sigma_{1} \# \Sigma_{2}\right) \cup \Sigma_{3} \cup \cdots \cup \Sigma_{m}$ still fills $M$.

Because $\Sigma_{3}$ intersects $\Sigma_{1} \cup \Sigma_{2}$, it intersects $\Sigma_{1} \# \Sigma_{2}$. Where $\Sigma_{1} \# \Sigma_{2}$ and $\Sigma_{3}$ intersect transversely there is a triple point of $\Sigma$ (and therefore of $\left.\left(\Sigma_{1} \# \Sigma_{2}\right) \cup \cdots \cup \Sigma_{m}\right)$. We can perform another piping operation (as before) obtaining a new Dehn sphere $\Sigma_{1} \# \Sigma_{2} \# \Sigma_{3}$ such that the new Dehn surface $\left(\Sigma_{1} \# \Sigma_{2} \# \Sigma_{3}\right) \cup \Sigma_{4} \cup \cdots \cup \Sigma_{m}$ still fills $M$.

Inductively, for $k>3$, we obtain a Dehn sphere $\Sigma_{1} \# \cdots \# \Sigma_{k}$ piping $\Sigma_{1} \# \cdots \# \Sigma_{k-1}$ with $\Sigma_{k}$ around a triple point of $\Sigma$ lying in the intersection of $\Sigma_{1} \# \cdots \# \Sigma_{k-1}$ and $\Sigma_{k}$, with the property that $\left(\Sigma_{1} \# \cdots \# \Sigma_{k}\right) \cup \Sigma_{k+1} \cup$ $\cdots \cup \Sigma_{m}$ still fills $M$.

Repeating this operation we finally obtain a Dehn sphere $\Sigma^{\prime}=\Sigma_{1} \#$ $\cdots \# \Sigma_{m}$ that fills $M$.

If all components of $\Sigma$ are nullhomotopic, this implies that we can deform the Dehn sphere $\Sigma_{m}$ continuously to a point. If $g_{m}: S^{2} \rightarrow M$ is an immersion parametrizing $\Sigma^{\prime}$, we can use this deformation to construct a homotopy between $g_{m}$ and an immersion $g_{m-1}$ parametrizing $\Sigma_{1} \# \cdots \# \Sigma_{m-1}$. In the same way, we can construct a homotopy between $g_{m-1}$ and an immersion $g_{m-2}$ parametrizing $\Sigma_{1} \# \cdots \# \Sigma_{m-2}$. Repeating this process, we finally conclude that $g_{m}$ is homotopic to an immersion $g_{1}$ parametrizing $\Sigma_{1}$ and so $g_{m}$ is nullhomotopic. 


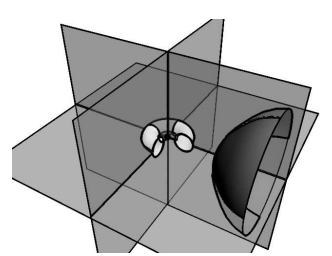

(a)

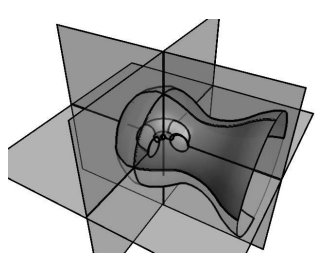

(b)

Fig. 6

Another property of spiral pipings is that they do not disturb filling homotopies, as stated in the following lemma, which we give without proof:

Lemma 15. Let $f, g: S \rightarrow M$ be two filling immersions such that $g$ is obtained from $f$ after a finger move +2 through the triple point $P$ of $f$. Let $S^{\prime}, f^{\prime}, g^{\prime}$ be the surface and immersions that come from $S, f, g$ respectively after performing a spiral piping around $P$. Assume that this spiral piping is as small as necessary, in comparison with the finger move (Figure 6(b)). Then $f^{\prime}$ and $g^{\prime}$ are filling homotopic.

In the situation of this lemma we say that the immersions $f^{\prime}, g^{\prime}$ are related by a piping passing move through $P$.

6. What can be done using filling-preserving moves. We will give two examples of operations in a filling Dehn surface using only fillingpreserving moves.

Let $\Sigma$ be a filling Dehn surface of the 3-manifold $M$.

6.1. Inflating a double point. Let $P$ be a double point of $\Sigma$. Consider a standardly embedded 2-sphere $\Sigma_{P}$ in $M$ as in Figure 7(b). It contains $P$,

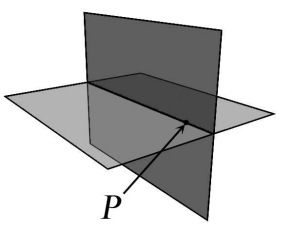

(a)

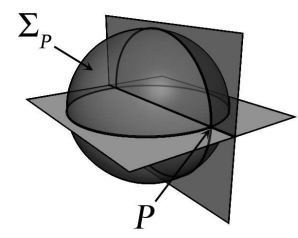

(b)

Fig. 7

and its intersection with $\Sigma$ is the union of two circles. The circles intersect at $P$ and at another point $Q$; these are the unique double points of $\Sigma$ lying in $\Sigma_{P}$. Note that $\Sigma \cup \Sigma_{P}$ is a filling Dehn surface of $M$. Consider a filling Dehn surface $\Sigma \# \Sigma_{P}$ obtained by modifying $\Sigma \cup \Sigma_{P}$ by a spiral piping around $P$ (see Section 5).

Proposition 16. We can choose the piping such that $\Sigma$ is filling homotopic to $\Sigma \# \Sigma_{P}$. 
Proof. We consider the filling Dehn surface $\Sigma \# \Sigma_{P}$ as in Figure 8(a). This surface is identical with $\Sigma \cup \Sigma_{P}$ except in a small neighbourhood of $P$, where it looks like Figure 8(b).

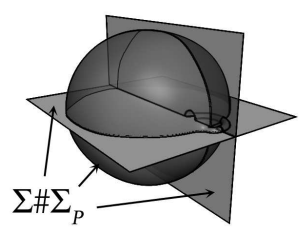

(a)

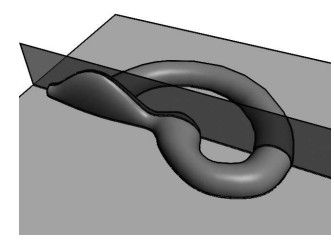

(c)

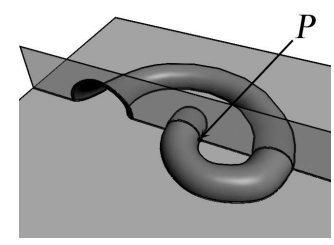

(e)

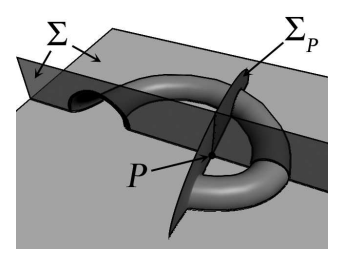

(b)

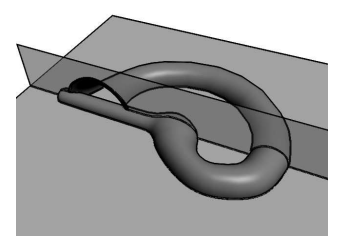

(d)

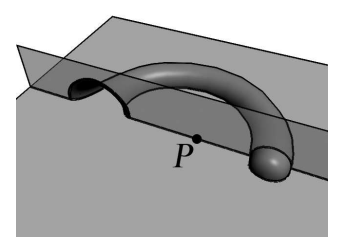

(f)

Fig. 8

We can uninflate $\Sigma_{P}$ through $P$ by an ambient isotopy of $M$, until we reach the situation depicted in Figure 8(c).

We "open the entrance of the tunnel" using two filling-preserving saddle moves, over and under the sheet of $\Sigma$ containing the spiral piping (Figure $8(\mathrm{c})$ ), and we get the situation of Figure 8(d). Now, after three consecutive finger moves -1 we make $\Sigma_{P}$ disappear completely (Figures $8(\mathrm{e})$ and $8(\mathrm{f})$ ).

In the above proposition, the statement could be "every parametrization of $\Sigma$ is filling homotopic to a parametrization of $\Sigma \# \Sigma_{P}$ ", which is a little stronger, but we use the above language for simplicity.

We say that the Dehn surface $\Sigma \# \Sigma_{P}$ as in the proposition is obtained from $\Sigma$ by inflating $P$.

6.2. Passing over 3-cells. Let $R$ be a regular region of $M-\Sigma$ such that each cell of $\partial R$ is also regular. In this case, we say that $R$ is $\partial$-regular. Let $\Delta$ be a face of $R$. The $\partial$-regularity of $R$ implies that $\operatorname{cl}(\Delta)$ is a closed disk and $\operatorname{cl}(R)$ is a closed 3-ball. Take a parametrization $f: S \rightarrow M$ of $\Sigma$, and 
take the immersion $g: S \rightarrow M$ obtained from $f$ by a pushing disk $(D, B)$ as indicated in Figure 9. There is an open disk $\widetilde{\Delta}$ in $S$ such that the restriction

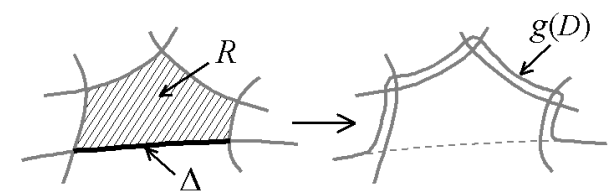

Fig. 9

of $f$ to $\widetilde{\Delta}$ is an embedding and $f(\widetilde{\Delta})=\Delta$. The pushed disk $D$ in $S$ contains $\operatorname{cl}(\widetilde{\Delta})$ in its interior and it is as close to $\widetilde{\Delta}$ as necessary, so that $\left.f\right|_{D}$ is an embedding. The pushing ball $B$ contains $R$, and $\operatorname{cl}(R) \cap \partial B=\operatorname{cl}(\Delta)$. The disk $g(D)$ is a closed disk outside $\operatorname{cl}(R)$ running in parallel to $\partial R-\Delta$. In [V2] the following is proved:

Lemma 17. If $g$ is a filling immersion, then it is filling homotopic to $f$.

7. Shellability. Smooth triangulations. In the proof of Theorem 2 we make an exhaustive use of the concept of shelling.

Definition 18. Let $N$ be an $n$-manifold with boundary, and let $C \subset N$ be a closed $n$-ball in $N$. We say that $C$ is free in $N$ if $C \cap \partial N$ is a closed $(n-1)$-ball.

Let $B$ be a closed $n$-ball, and let $K$ be a regular cell decomposition of $B$.

Definition 19. $K$ is shellable if there exists an ordering $C_{1}, \ldots, C_{k}$ of its $n$-cells such that $\operatorname{cl}\left(C_{i}\right)$ is free in the closure of $\bigcup_{j \geq i} C_{j}$. If this is the case, we say that the ordering $C_{1}, \ldots, C_{k}$ is a shelling of $\bar{K}$.

While cell decompositions of 2-disks are always shellable (see Lemma 1 of $[\mathrm{S}]$ ), nonshellable cell decompositions of $n$-balls exist for $n>2$. In [B-M] it is proved that every cell decomposition of an $n$-ball has a shellable subdivision (a cell decomposition $\sigma K$ of $B$ is a subdivision of the cell decomposition $K$ of $B$ if every cell of $\sigma K$ is contained in a cell of $K$ ).

For the proof of Theorem 2, we will work with triangulations of a 3manifold $M$ and we will require that a set of not necessarily disjoint 3-balls in $M$ (endowed with the induced cell decomposition) are all shellable at once, and with some special properties. For this, we will use the work of Whitehead about simplicial collapsings (see [Wh1] or [Gl]).

A simplicial complex whose underlying polyhedron is a ball induces in a natural way a cell decomposition of the ball. Thus, we can consider simplicial complexes also as particular cell complexes. 
If $K$ is a simplicial complex, a simplex of $K$ is maximal if it is not a proper face of another simplex of $K$. If $\varepsilon^{i}$ is a maximal $i$-simplex of $K$, an $(i-1)$-face $\varepsilon^{i-1}$ of $\varepsilon^{i}$ is free in $K$ if it is not a face of another $i$-simplex of $K$ different from $\varepsilon^{i}$. If $\varepsilon^{i}$ is maximal in $K$ and $\varepsilon^{i-1}$ is free in $K$, then the result of removing $\left(\varepsilon^{i}, \varepsilon^{i-1}\right)$ from $K$ is another simplicial complex $K^{\prime}$, said to be obtained from $K$ by a simplicial collapsing. The complex $K$ collapses simplicially into a subcomplex $K^{\prime}$ if $K^{\prime}$ is obtained from $K$ after a finite sequence of simplicial collapsings. In particular, $K$ is collapsible if it collapses simplicially into a point. If $\sigma K$ is a subdivision of $K$ and $K^{\prime}$ is a subcomplex of $K$, then $\sigma K^{\prime}$ will denote the corresponding subcomplex of $\sigma K$.

TheOREM 20 ([Wh1]). If $K$ is any finite simplicial complex, there is $a$ (stellar) subdivision $\sigma K$ of $K$ such that $\sigma B^{n}$ collapses simplicially into $\sigma B^{n-1}$, where $B^{n}$ is any subcomplex of $K$ which is a closed $n$-ball and $B^{n-1}$ is any subcomplex of $\partial B^{n}$ which is a closed $(n-1)$-ball.

For triangulations of an $n$-ball, shellability obviously implies collapsibility. Note that the converse is not obvious because in shellability we require that the space after each step remains a ball, while in "collapsings" it might not even be a manifold. (The example given in $[\mathrm{Ru}]$ is not shellable, but it is simplicially collapsible, cf. [Ch].) However, the converse is almost true in our case according to the following observation that arises from the proof of Theorem 6 in $[\mathrm{Bi}]$ :

Lemma 21. If $K$ is a collapsible triangulation of the 3 -ball, then the second derived subdivision of $K$ is shellable.

Smooth triangulations of manifolds are introduced in [Wh2] to relate the smooth and PL categories in manifold theory. A triangulation of an $n$-manifold $N$ is a homeomorphism $h: K \rightarrow N$, where $K$ is a rectilinear simplicial complex of some euclidean space. If $N$ has a smooth structure, the triangulation $h$ is smooth (with respect to this structure) if the restriction of $h$ to each simplex of $K$ is a smooth map. We identify the manifold $N$ with the simplicial complex $K$. In [Wh2] (see also [Mu]) it is proved that: (i) any $n$-manifold with a smooth structure admits smooth triangulations; and (ii) two smooth triangulations of the same smooth manifold have a common smooth subdivision (!). If $f: S \rightarrow M$ is a transverse immersion of a surface into the 3 -manifold $M$, then there are smooth triangulations $K$ and $T$ of $S$ and $M$ respectively such that $f$ is simplicial with respect to them (for more general results of this kind, see [Ve]).

If $f: S \rightarrow M$ is a filling immersion and $K, T$ are triangulations of $S, M$ respectively such that $f$ is simplicial with respect to them, then the triangulation $T$ also triangulates the closure of each region of $M-f(S)$. If $R$ is a regular region of $M-f(S)$, we say that $T$ shells $R$ if it induces a shellable 
triangulation on $\operatorname{cl}(R)$. If $R$ is not regular, we first cut $\operatorname{cl}(R)$ along its selfadjacencies to obtain a closed 3-ball $\mathrm{cl}(R)$. The triangulation $T$ on $\operatorname{cl}(R)$ lifts naturally to $\widetilde{\operatorname{cl}(R)}$, and we say that $T$ shells $R$ if the induced triangulation on $\widetilde{\operatorname{cl}(R)}$ is shellable. The triangulation $T$ shells the filling immersion $f$ (or the filling Dehn surface $f(S))$ if $T$ shells each region of $M-f(S)$.

All these results imply the following

Theorem 22. Let $S_{1}, \ldots, S_{k}$ be a finite collection of surfaces, and for each $i=1, \ldots, k$ let $f_{i}: S_{i} \rightarrow M$ be a transverse immersion. Then there is a smooth triangulation $T$ of $M$ such that:

(1) the Dehn surfaces $f_{1}\left(S_{1}\right), \ldots, f_{k}\left(S_{k}\right)$ are contained in the 2 -skeleton of $T$;

(2) if $f_{i}$ is a filling immersion for some $i=1, \ldots, k$, then the triangulation $T$ shells $f_{i}$;

(3) if $f_{i}$ and $f_{j}$ differ by a pushing disk $(D, B)$ for some $i, j \in\{1, \ldots, k\}$, then the triangulation $T$ restricted to $B$ collapses simplicially into $f_{j}(D)$.

Proof. First of all, we have seen that for each $i=1, \ldots, k$ there are smooth triangulations $K_{i}, T_{i}$ such that $f_{i}$ is simplicial with respect to them. According to [Wh2], the smooth triangulations $T_{1}, \ldots, T_{k}$ have a common smooth subdivision $T_{0}$. Then all the Dehn surfaces $f_{1}\left(S_{1}\right), \ldots, f_{k}\left(S_{k}\right)$ are contained in the 2-skeleton of $T_{0}$. Take a subdivision $T_{0}^{\prime}$ of $T_{0}$ as in Theorem 20 , and let $T$ be the second derived subdivision of $T_{0}^{\prime}$.

If $f_{i}$ and $f_{j}$ differ by a pushing disk $(D, B)$, then $T_{0}$ triangulates $B$ and $f_{j}(D)$, and so because $T_{0}^{\prime}$ has been chosen following Theorem 20 , the triangulation $T_{0}^{\prime}$ restricted to $B$ collapses simplicially into $f_{j}(D)$. Simplicial collapsing is preserved by stellar subdivisions [Wh1] and so it is also preserved by derived subdivisions. Thus, $T$ restricted to $B$ collapses simplicially into $f_{j}(D)$.

If $f_{i}$ is a filling immersion and $R$ is a regular region of $M-f_{i}\left(S_{i}\right)$, then $T_{0}$ triangulates $\mathrm{cl}(R)$. By the choice of $T_{0}^{\prime}$, it induces a collapsible triangulation of $\operatorname{cl}(R)$, and by Lemma 21, $T$ induces a shellable triangulation of $\operatorname{cl}(R)$. If $R$ is not regular, perhaps we need to do more stellar subdivisions on $\operatorname{cl}(R)$ to have the required shelling property on $\widetilde{\mathrm{cl}(R)}$, but this does not alter the previous construction because stellar subdivisions preserve shellability [B-M] and collapsibility.

Definition 23. Under the hypothesis of the previous theorem, we say that $T$ is a good triangulation of $M$ with respect to $f_{1}, \ldots, f_{k}$.

With these results, we have prepared the ground for the following sections. 
8. Inflating triangulations. Now we will explain how we can associate to any triangulation of the 3-manifold $M$ a filling collection of spheres of $M$.

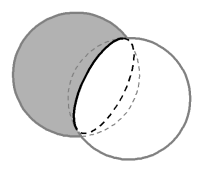

(a)

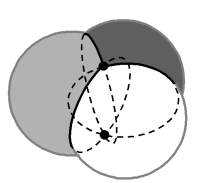

(b)

Fig. 10

Let $B_{1}, B_{2}$ be two closed 3 -balls in $M$. We say that $B_{1}, B_{2}$ intersect normally if they intersect as in Figure 10(a). The 2-spheres $\partial B_{1}, \partial B_{2}$ must intersect transversely in a unique simple closed curve. If $B_{1}, B_{2}$ intersect normally, then $B_{1} \cap B_{2}, \operatorname{cl}\left(B_{1}-B_{2}\right)$ and $\mathrm{cl}\left(B_{2}-B_{1}\right)$ are 3 -balls. If $B_{1}, B_{2}, B_{3}$ are 3-balls in $M$, they intersect normally if they intersect as in Figure 10(b). Each pair $B_{i}, B_{j}$ with $i \neq j$ intersect normally and $\partial B_{1}, \partial B_{2}$ and $\partial B_{3}$ intersect transversely at two triple points.

Let $T$ be a smooth triangulation of $M$. (We refer to the 0 -simplexes, 1-simplexes, 2-simplexes and 3-simplexes of $T$ as vertices, edges, triangles and tetrahedra of $M$, respectively.) We can construct a filling collection of spheres of $M$ by "inflating" $T$ assigning to each simplex $\varepsilon$ of the 2-skeleton $T^{2}$ of $T$ a 2 -sphere $S \varepsilon$ embedded in $M$ in such a way that their union $\mathbb{T}=\bigcup_{\varepsilon \in T^{2}} S \varepsilon$ fills $M$. We will do this as follows.

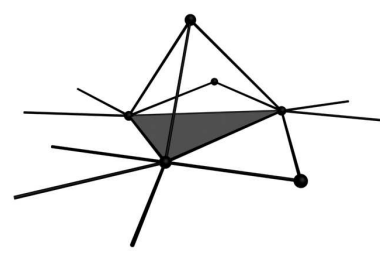

(a)

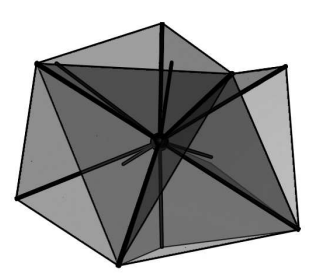

(c)

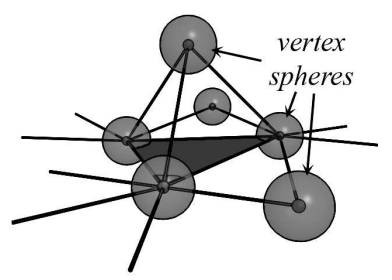

(b)

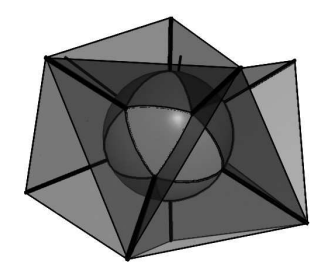

(d)

Fig. 11

First, if $v_{1}, \ldots, v_{m_{0}}$ are the vertices of $M$, for $i=1, \ldots, m_{0}$ the 2 -sphere $S v_{i} \subset M$ bounds a closed 3-ball $B v_{i}$ in $M$ contained in the open $\operatorname{star} \operatorname{star}\left(v_{i}\right)$ 
and with $v_{i}$ in its interior. The 2-spheres $S v_{1}, \ldots, S v_{m_{0}}$ are pairwise disjoint (Figure 11(b)) and the triangulation $T$ of $M$ induces a triangulation of $B v_{i}$ as a cone from $v_{i}$ over $S v_{i}$ for each $i=1, \ldots, m_{0}$ (see Figure 11(d)). The 2-sphere $S v_{i}$ intersects transversely each $i$-simplex $\varepsilon^{i} \in \operatorname{star}\left(v_{i}\right) \subset T$ in a $(i-1)$-simplex of this induced triangulation of $S v_{i}$.

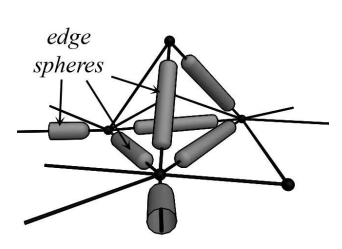

(a)

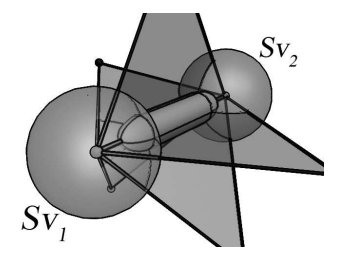

(c)

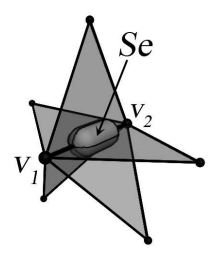

(b)

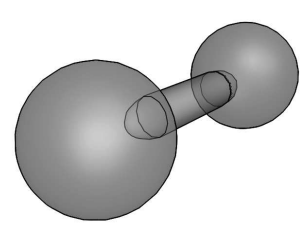

(d)

Fig. 12

If $e_{1}, \ldots, e_{m_{1}}$ are the edges of $M$, for $j=1, \ldots, m_{1}$ the 2 -sphere $S e_{j} \subset M$ bounds a closed 3 -ball $B e_{j}$ in $M$ as in Figure 12(a). The 3 -ball $B e_{j}$ is contained in the open star $\operatorname{star}\left(e_{j}\right)$ and it intersects $e_{j}$ in a closed subarc $\widetilde{e}_{j} \subset e_{j}$. The 2 -sphere $S e_{j}$ and $e_{j}$ intersect transversely at the endpoints of the arc $\widetilde{e}_{j}$. We take $B e_{1}, \ldots, B e_{m_{1}}$ pairwise disjoint, and for each $i \in$ $\left\{1, \ldots, m_{0}\right\}$ and $j \in\left\{1, \ldots, m_{1}\right\}$ the 3 -balls $B v_{i}$ and $B e_{j}$ are also disjoint unless $v_{i}$ and $e_{j}$ are incident. In this case, $B v_{i}$ and $B e_{j}$ intersect normally (Figure 12(c)) and $B v_{i} \cap B e_{j}$ intersects $e_{j}$ in another closed subarc of $e_{j}$. Considering the two points of the intersection $S e_{j} \cap e_{j}$ as the "poles" of $S e_{j}$, each triangle $t$ of $M$ incident with $e_{j}$ intersects $S e_{j}$ transversely in an open arc which is the interior of a "meridian" $a$ with its endpoints at the poles (Figure 12(b)). The intersection $\operatorname{cl}(t) \cap B e_{j}$ is a closed disk bounded by $a \cup \widetilde{e}_{j}$.

Finally, if $t_{1}, \ldots, t_{m_{2}}$ are the triangles of $M$, for $k=1, \ldots, m_{2}$ the 2sphere $S t_{k}$ bounds a 3 -ball $B t_{k}$ as in Figure 13. The 3 -ball $B t_{k}$ is contained in the (open) star $\operatorname{star}\left(t_{k}\right)$ and it intersects $t_{k}$ in a closed disk $\widetilde{t}_{k} \subset t_{k}$, and the intersection of $S t_{k}$ with $t_{k}$ is transverse. The 3-ball $B t_{k}$ is disjoint from $B \varepsilon$ for every $\varepsilon \in T^{2}$ different from $t_{k}$ unless $\varepsilon$ is incident with $t_{k}$. In this case, $B t_{k}$ and $B \varepsilon$ intersect normally. Moreover, if $v_{i}<e_{j}<t_{k}$, then the 3-balls $B v_{i}, B e_{j}, B t_{k}$ intersect normally (Figure 13) and there is one of the two triple points of $S v_{i} \cap S e_{j} \cap S t_{k}$ in each of the two tetrahedra of $\operatorname{star}\left(t_{k}\right)$. 


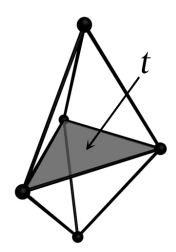

(a)

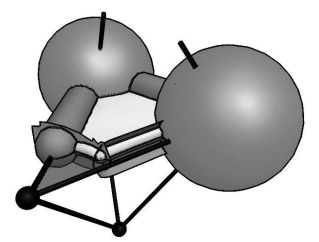

(c)

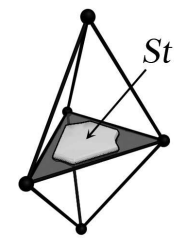

(b)

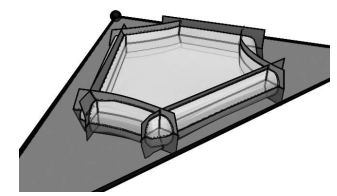

(d)

Fig. 13

If $T$ is a cell decomposition of $M$ instead of a triangulation, the previous construction can easily be generalized.

It is easy to check that the Dehn surface $\mathbb{T}=\bigcup_{\varepsilon \in T^{2}} S \varepsilon$ so constructed is a filling collection of spheres in $M$. Moreover, $\mathbb{T}$ is regular and it is transverse to the (smooth) simplexes of the triangulation $T$ of $M$.

In particular, as a corollary of Theorem 14 this construction implies the main theorem of [Mo2]:

Theorem 24. $M$ has a nullhomotopic filling Dehn sphere.

Note that in this case, in contradistinction to [Mo2] or [V1], we have not made any assumption about the orientability of $M$.

The following result follows directly from the construction.

Proposition 25. Let $S$ be a surface and $f: S \rightarrow M$ a transverse immersion. Let $K, T$ be triangulations of $S, M$ respectively such that $f$ is simplicial with respect to them. Then $f(S) \cup \mathbb{T}$ is a regular filling surface of $M$.

In this proposition the immersion $f$ can be any transverse immersion, filling or not. Assume now that $f: S \rightarrow M$ is a filling immersion and put $\Sigma:=f(S)$. Let $K, T$ be triangulations of $S$ and $M$ respectively such that $f$ is simplicial with respect to them. By Proposition $25, \Sigma \cup \mathbb{T}$ fills $M$, and by the methods of proof of Theorem 14, we can obtain from $\Sigma \cup \mathbb{T}$ a unique filling Dehn surface of $M$. If we look at the proof of Theorem 14, this can be done in many different ways because there are many possibilities for performing the spiral pipings. We say that each filling Dehn surface $\Sigma^{\prime}$ of $M$ that is obtained from $\Sigma$ in this way is a T-inflating of $\Sigma$. Let $\Sigma^{\prime}$ be a $T$-inflating of $\Sigma$. By Propositions 25 and $12, \Sigma^{\prime}$ is regular because spiral pipings preserve regularity. There is an immersion $f^{\prime}: S \rightarrow M$ parametrizing 
$\Sigma^{\prime}$ that comes from $f$ in a natural way, that is, $f^{\prime}$ agrees with $f$ in most of $S$ except in the small disks where we perform the pipings. We also say that $f^{\prime}$ is a $T$-inflating of $f$. The first application of shellability is the next result proved in [V2].

Proposition 26. If $T$ shells $f$, then there is a T-inflating $f^{\prime}$ of $f$ filling homotopic to $f$. Moreover, we can choose $f^{\prime}$ such that there are only two spiral pipings connecting $\Sigma$ with components of $\mathbb{T}$ and the other spiral pipings are performed around triple points of $\mathbb{T}$.

By Theorem 22, passing to suitable subdivisions we can assume that $T$ shells $f$, and thus we have:

Corollary 27. If $f: S \rightarrow M$ is a filling immersion, then $f$ is filling homotopic to a regular filling immersion.

The proof of Proposition 26 is made by repeated application of the construction of Section 6.1, using the fact that each region of $M-\Sigma$ has a shellable triangulation and that each triangulation of a 2-disk is shellable [S]. As an example, we will illustrate the starting point of this construction in which we "inflate" a triangle of $T$.

EXAMPle 28 (Inflating a triangle). Let $f, \Sigma$ and $T$ be as in Proposition 26. Imagine that there exists a triangle $t$ of $T$ intersecting $\Sigma$ as in Figure 14. We label the edges and vertices of $t$ as in the figure.

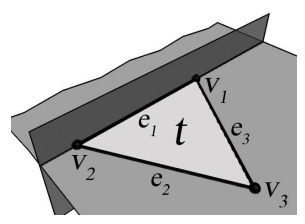

Fig. 14

Let $Q_{0}$ be the intersection point of the 2-sphere $S v_{1}$ with $e_{1}$ (Figure $15(\mathrm{~b}))$. We inflate $Q_{0}$ to obtain a 2 -sphere $\Sigma_{Q_{0}}$ connected with $\Sigma$ by a spiral piping as in Figure 15(a). Then, after ambient isotopy, we obtain the filling Dehn surface $\Sigma \# \Sigma_{Q_{0}}$, which is obtained from $\Sigma \cup \Sigma_{Q_{0}}$ by a spiral piping around $Q_{0}$.

Let $Q_{1}$ be the intersection point of $S e_{1}$ with $e_{1}$ that lies inside $B v_{1}$ (Figure 15(c)). We inflate it (Figure 15(d)). After a piping passing move (Figure 15(e)), we obtain a filling Dehn surface ambient isotopic to that of Figure 15(f).

Let $P_{1}$ be one of the two points of $S v_{1} \cap S e_{1} \cap S t$ (Figure 16(a)). This point is a double point of the filling Dehn surface of Figure 15(f), and thus we can inflate it using filling-preserving moves until we obtain the filling Dehn surface of Figure 16(b) (with a spiral piping around $P_{1}$ ). After a finger move 


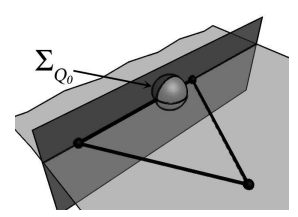

(a)

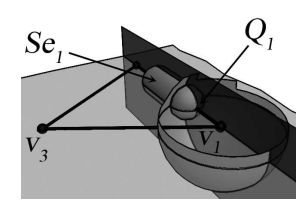

(c)

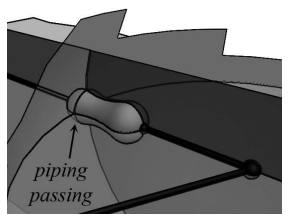

(e)

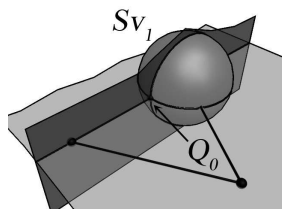

(b)

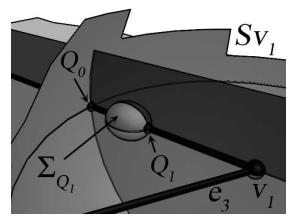

(d)

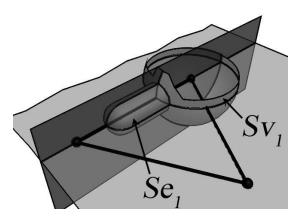

(f)

Fig. 15

+1 through the triple point $A$ of the same Figure 16(b), we get the filling Dehn surface of Figure 16(c), which is the same as that of Figure 16(d). The surfaces of Figures 16(a) and 16(d) are ambient isotopic.

At this moment, from the picture of Figure 14 we have obtained the 2-spheres $S v_{1}, S e_{1}$ and $S t$, with three spiral pipings, using only fillingpreserving moves. Using similar methods, we can also obtain the 2 -spheres $S v_{2}$ (Figure 16), $S e_{2}, S v_{3}$ and $S e_{3}$ (Figure 17).

In the previous example, we see that the way of constructing the filling Dehn surface $\Sigma^{\prime}$ from the filling Dehn surface $\Sigma$ in Proposition 26 is in some sense to make $\Sigma$ grow inductively following a path given by the triangulation $T$. The growing path we follow to prove Proposition 26 in [V2] is not exactly as in Example 28. There (in [V2]) we inflate first from $\Sigma$ all the 2 -spheres of $\mathbb{T}$ corresponding to the simplexes of $T$ contained in $\Sigma$ starting in a similar way to Example 28. Then the shellability conditions imposed on $T$ will give us the growing path of $\Sigma$ on the regions of $M-\Sigma$ using again similar methods to those of Example 28.

We will say that the $T$-inflating $\Sigma^{\prime}$ of $\Sigma\left(f^{\prime}\right.$ of $\left.f\right)$ as in the previous proposition is a $T$-growth of the filling Dehn sphere $\Sigma$ (of the filling immersion $f$ ). Note that to be a $T$-growth is stronger than to be a $T$-inflating.

The next (but not last) application of the constructions of Section 7 is the following. 


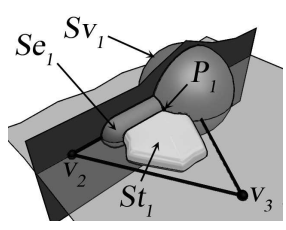

(a)

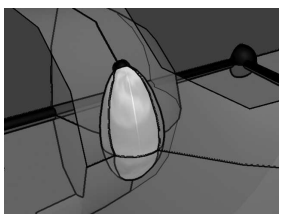

(c)

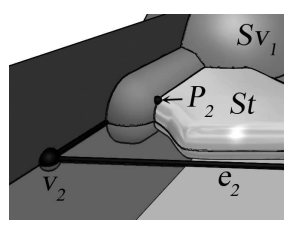

(e)

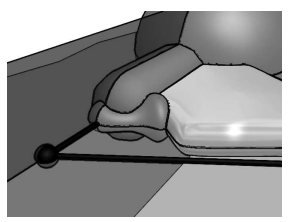

$(\mathrm{g})$

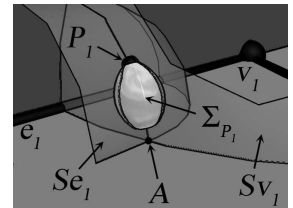

(b)

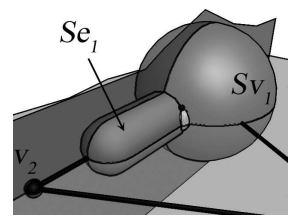

(d)

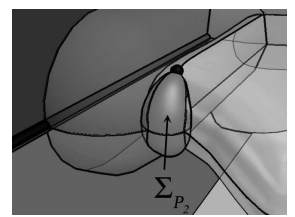

(f)

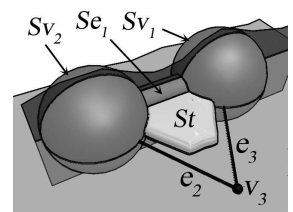

(h)

Fig. 16

Let $f, g: S \rightarrow M$ be transverse immersions that differ by the pushing disk $(D, B)$, and assume that $f$ is a filling immersion. In this situation, the immersion $g$ will not necessarily be a filling immersion. Consider triangulations $K, T$ of $S$ and $M$ respectively such that $f$ and $g$ are simplicial with respect to them. Take a $T$-inflating $f^{\prime}$ of $f$ such that $f^{\prime}$ agree with $f$ over $D$ (because $f^{\prime}$ agrees with $f$ in most of $S$ except in some small disks of $S$, we require that these small disks do not intersect $D$ ), and consider the immersion $g^{\prime}$ that is obtained from $f^{\prime}$ after the pushing disk $(D, B)$. We can assume that $g^{\prime}$ agrees with $g$ except in the disks of $S$ where $f^{\prime}$ "disagrees" with $f$. The Dehn surface $g^{\prime}(S)$ is obtained from $g(S) \cup \mathbb{T}$ by spiral pipings, and because $g$ is also simplicial (with respect to $K, T$ ), $g^{\prime}$ is a $T$-inflating of $g$.

Because both $f, g$ are simplicial (with respect to $K, T$ ), the triangulation $T$ induces a triangulation of the pushing ball $B$.

Lemma 29 (Key Lemma 2). If the (induced) triangulation of $B$ collapses simplicially into $g(D)$, then $f^{\prime}$ is filling homotopic to $g^{\prime}$. 


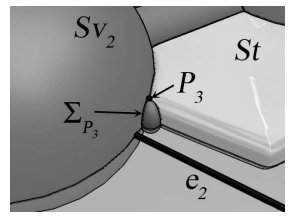

(a)

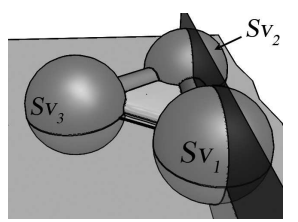

(d)

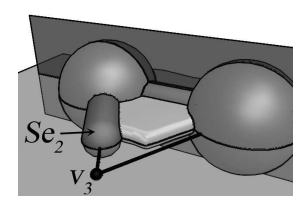

(b)

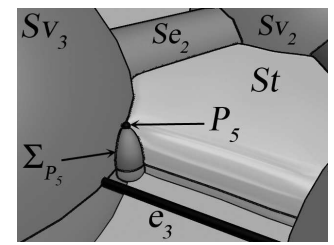

(e)

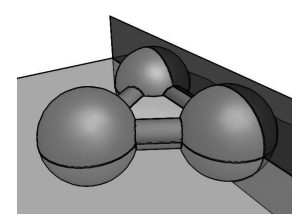

(g)

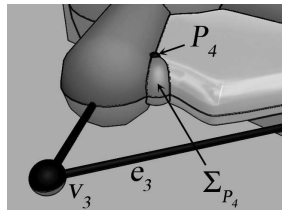

(c)

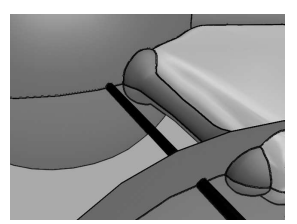

(f)

Fig. 17

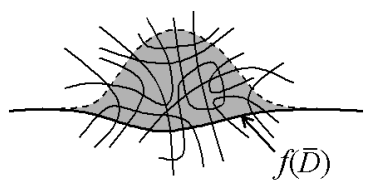

(a)

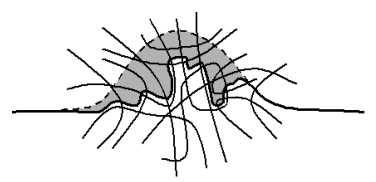

(b)

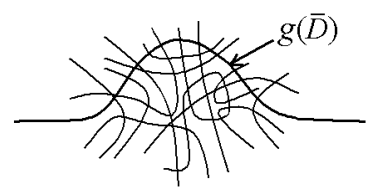

(c)

Fig. 18

Sketch of proof. Because $B$ is triangulated by $T$, it can be easily shown that $f(S) \cup g(S) \cup \mathbb{T}$ induces a cell decomposition of $B$. If $B$ collapses simplicially into $g(D)$, then we can define a special shelling of this cell decomposition. This special shelling will allow us to apply Lemma 17 repeatedly (Figure 18(b)) to the filling Dehn sphere $f(S) \cup \mathbb{T}$ until we get $g(S) \cup \mathbb{T}$. Substituting finger moves 2 by piping passing moves where required, this deformation of $f(S) \cup \mathbb{T}$ into $g(S) \cup \mathbb{T}$ also defines a deformation of $f^{\prime}$ into $g^{\prime}$ by filling-preserving moves. 
9. Filling pairs. Let $\Sigma_{1}$ and $\Sigma_{2}$ be two filling Dehn surfaces of $M$. Assume by simplicity that both are regular.

If we are not given more information about $\Sigma_{1}$ and $\Sigma_{2}$, we do not know how they are related to each other. The only thing we can say, if $M$ is not $S^{3}$, is that they must have nonempty intersection.

Definition 30. We say that $\Sigma_{1}$ and $\Sigma_{2}$ form a filling pair in $M$ if their union $\Sigma_{1} \cup \Sigma_{2}$ is also a regular filling Dehn surface of $M$.

In particular, if $\Sigma_{1}$ and $\Sigma_{2}$ form a filling pair in $M$, they intersect transversely.

If $\Sigma_{1}$ and $\Sigma_{2}$ are a filling pair in $M$, then $\Sigma_{2}$ induces a cell decomposition on the closure of each region of $M-\Sigma_{1}$ and vice versa. Because both $\Sigma_{1}$ and $\Sigma_{2}$ are regular, all these induced cell decompositions are also regular. If $R_{1}$ is a region of $M-\Sigma_{1}$, then we say that $\Sigma_{2}$ shells $R_{1}$ if $\Sigma_{2}$ induces a shellable cell decomposition of the 3 -ball $\mathrm{cl}\left(R_{1}\right)$. We say that $\Sigma_{2}$ shells $\Sigma_{1}$ if $\Sigma_{2}$ shells each region of $M-\Sigma_{1}$.

Definition 31. Let $\Sigma_{1}$ and $\Sigma_{2}$ form a filling pair in $M$. We say that $\Sigma_{1}$ and $\Sigma_{2}$ are mutually shellable if $\Sigma_{1}$ shells $\Sigma_{2}$ and $\Sigma_{2}$ shells $\Sigma_{1}$.

The following result is proved in detail in [V2].

Proposition 32. Let $\Sigma_{1}, \Sigma_{2}$ be regular filling Dehn surfaces of $M$ which intersect transversely. If $f_{1}: S_{1} \rightarrow M$ parametrizes $\Sigma_{1}$, then $f_{1}$ is filling homotopic to an immersion $f_{1}^{\prime}: S_{1} \rightarrow M$ such that $\Sigma_{1}^{\prime}:=f^{\prime}\left(S_{1}\right)$ and $\Sigma_{2}$ form a mutually shellable filling pair in $M$.

Sketch of proof. Let $f_{2}: S_{2} \rightarrow M$ be a parametrization of $\Sigma_{2}$, and let $T$ be a good triangulation of $M$ with respect to $f_{1}$ and $f_{2}$ (Definition 23). Then $T$ shells every region of $M-\Sigma_{1}$ and every region of $M-\Sigma_{2}$. The union $\Sigma_{1} \cup \Sigma_{2} \cup \mathbb{T}$ is a regular filling Dehn surface of $M$ by Proposition 25 . Take a $T$-growth $f_{1}^{\prime}$ of $f_{1}$, and put $\Sigma_{1}^{\prime}=f_{1}^{\prime}\left(S_{1}\right)$. We make the spiral pipings that transform $\Sigma_{1} \cup \mathbb{T}$ into $\Sigma_{1}^{\prime}$ small enough not to intersect $\Sigma_{2}$. Because regularity is preserved by spiral pipings, it is not difficult to see that $\Sigma_{1}^{\prime} \cup \Sigma_{2}$ is a regular filling Dehn surface of $M$. It is also easy to see that $\Sigma_{2}$ induces a shellable cell decomposition on every region of $M-\Sigma_{1}^{\prime}$ using the fact that $\Sigma_{2}$ is a subcomplex of $T$ and the construction of $\mathbb{T}$. The nontrivial part is to check that $\Sigma_{1}^{\prime}$ induces a shellable cell decomposition on every region of $M-\Sigma_{2}$. This is done in detail in [V2], and it is parallel to the proof of Key Lemma 2 above. If $R_{2}$ is a region of $M-\Sigma_{2}$, the first thing to check is that $\mathbb{T}$ induces a shellable cell decomposition on $\operatorname{cl}\left(R_{2}\right)$. This is done following the proof of Lemma 21 in [Bi], using the fact that the restriction to $\mathrm{cl}\left(R_{2}\right)$ of the triangulation $T$ is simplicially collapsible. After this, it is seen that the presence of $\Sigma_{1}$ does not alter this shellability property. Finally, the presence of the spiral pipings might affect the shelling in some cases. In [V2] it is 
explained how this can occur, and how we can choose $f_{1}^{\prime}$ to ensure that it satisfies the statement of Proposition 32.

10. Simultaneous growings. The last application of shellability will be the following. Assume that $\Sigma_{1}, \Sigma_{2}$ are two regular filling Dehn surfaces of $M$ and that there are two points $P, Q$ where $\Sigma_{1}$ and $\Sigma_{2}$ intersect as in Figure 19(a). We denote by $\Sigma_{1} \# \Sigma_{2}$ the Dehn surface of $M$ that arises by piping $\Sigma_{1}$ with $\Sigma_{2}$ near $P, Q$ as in Figure $19(\mathrm{~b})$. We also assume that the points

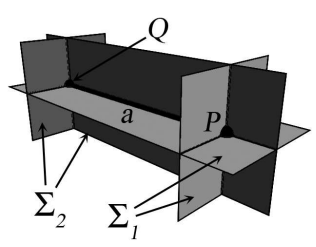

(a)

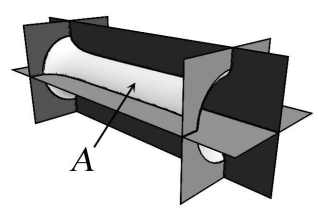

(b)

Fig. 19

$P, Q$ have the property that $\Sigma_{1} \# \Sigma_{2}$ is another filling Dehn surface of $M$. Let $f: S_{1} \rightarrow M, g: S_{2} \rightarrow M$ be parametrizations of $\Sigma_{1}, \Sigma_{2}$ respectively. Consider the two small disks $\delta_{1}, \delta_{2}$ of $S_{1}$ and $S_{2}$ respectively whose respective images under $f$ and $g$ disappear after the piping. In this situation we can construct a parametrization $f \# g: S_{1} \# S_{2} \rightarrow M$ of $\Sigma_{1} \# \Sigma_{2}$ "coming" from $f, g$, where the surface $S_{1} \# S_{2}$ is the result of identifying $S_{1}-\delta_{1}$ and $S_{2}-\delta_{2}$ along the boundary of $\delta_{1}$ and $\delta_{2}$. We can also assume that the immersion $f \# g$ agrees with $f$ on $S_{1}-\delta_{1}$ and that $f \# g$ agrees with $g$ on $S_{2}-\delta_{2}$.

Let $K_{1}, K_{2}, T$ be triangulations of $S_{1}, S_{2}, M$ that make $f, g$ and $f \# g$ simplicial, and assume that $T$ shells every region of $M-\Sigma_{1}$. Consider a $T$-growth $f^{\prime}$ of $f$ as in Proposition 26, such that $f^{\prime}$ agrees with $f$ in all of $S_{1}$ except in two small disks. We can assume that the images under $f$ of the two disks are far away from a sufficiently large regular neighbourhood of the arc $a$ of Figure 19(a) (that is, they do not affect the piping between $\Sigma_{1}$ and $\Sigma_{2}$ ). In this situation, we can also consider the "piped immersion" $f^{\prime} \# g: S_{1} \# S_{2} \rightarrow M$ that agrees with $f^{\prime}$ in $S_{1}-\delta_{1}$ and with $g$ in $S_{2}-\delta_{2}$, as the result of pasting $f^{\prime}$ and $g$ by means of the piping in exactly the same way as $f$ was pasted with $g$ in $f \# g$.

REMARK 33. If $\Sigma_{1}, \Sigma_{2}$ are the surfaces $\Sigma_{1}^{\prime}, \Sigma_{2}$ that result from the proof of Proposition 32, then there always exists a pair of points $P, Q$ as above.

We know that $f^{\prime}$ is filling homotopic to $f$ because it is a T-growth of $f$, but we also have:

Lemma 34 (Key Lemma 3). If $\Sigma_{2}$ shells $\Sigma_{1}$, then we can choose $f^{\prime}$ such that $f^{\prime} \# g$ is a T-growth of $f \# g$. 
In particular, if $\Sigma_{2}$ shells $\Sigma_{1}$, then we can choose $f^{\prime}$ such that $f^{\prime} \# g$ is filling homotopic to $f^{\prime}$.

This lemma is also proved in detail in [V2]. The required property that $\Sigma_{2}$ shells $\Sigma_{1}$ implies that the growing of $f$ into $f^{\prime}$ in the proof of Proposition 26 can be adapted to $\Sigma_{2}$ in such a way that the growing from $f$ into $f^{\prime}$ defines simultaneously a growing from $f \# g$ into $f^{\prime} \# g$ when we introduce $\Sigma_{2}$.

11. Proof of Theorem 2. With these tools we can sketch the proof of Theorem 2.

Sketch of proof of Theorem 2. We are given a pair $\Sigma_{1}, \Sigma_{2}$ of nullhomotopic filling Dehn spheres of $M$ and two parametrizations $f, g$ of them respectively. We introduce the following notation: we take two different 2spheres $S_{1}, S_{2}$ and we will consider that $S_{i}$ is the domain of $\Sigma_{1}$ for $i=1,2$. In particular, $\Sigma_{1}=f\left(S_{1}\right)$ and $\Sigma_{2}=g\left(S_{2}\right)$.

Modifying $f$ if necessary by an ambient isotopy of $M$ we can assume that $\Sigma_{1}$ and $\Sigma_{2}$ have nonempty transverse intersection.

By Proposition 32 and Remark 33, we can assume that $\Sigma_{1}, \Sigma_{2}$ form a mutually shellable filling pair of spheres of $M$ and that there are two points $P, Q$ of $\Sigma_{1} \cup \Sigma_{2}$ where $\Sigma_{1}$ and $\Sigma_{2}$ intersect as in Figure 19(a) of Section 10.

Consider the filling Dehn surface $\Sigma_{1} \# \Sigma_{2}$ and the parametrization $f \# g$ as in Section 10. We also consider the disks $\delta_{1} \subset S_{1}$ and $\delta_{2} \subset S_{2}$ as in Section 10. We denote $f \# g$ by $h$ for simplicity.

$h$ is filling homotopic to $f$.

Consider a small standardly embedded 2 -sphere $\Sigma_{2}^{*}$ and a parametrization $g_{*}: S_{2} \rightarrow M$ of $\Sigma_{2}^{*}$ as in Figure 20(b). This sphere shares with $\Sigma_{2}$ a 2-disk $\bar{D}$ containing $g\left(\delta_{2}\right)$ in its interior, and the immersions $g$ and $g_{*}$ agree over $\widetilde{D}:=g_{*}^{-1}(\bar{D})$.

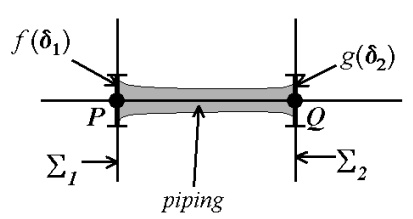

(a)

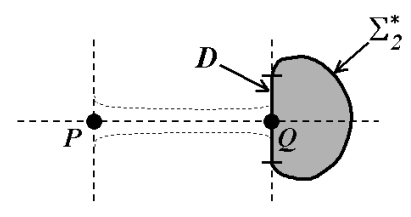

(b)

Fig. 20

By Key Lemma 1 , we can deform $g$ into $g_{*}$ by a finite sequence of transverse pushing disks leaving $\widetilde{D}$ fixed. Let $\left(D_{1}, B_{1}\right), \ldots,\left(D_{k}, B_{k}\right)$ be this sequence of pushing disks, and let $g=g_{0}, g_{1}, \ldots, g_{k}=g_{*}: S_{2} \rightarrow M$ be the sequence of transverse immersions such that $g_{i}$ is obtained from $g_{i-1}$ by the pushing disk $\left(D_{i}, B_{i}\right)$. 
Modifying slightly $\Sigma_{1}$ and the piping between $\Sigma_{1}$ and $\Sigma_{2}$ by an ambient isotopy of $M$ if necessary, we can assume that all these pushing disks are also transverse to $\Sigma_{1}$ and $\Sigma_{1} \# \Sigma_{2}$. Because the pushing disks $\left(D_{i}, B_{i}\right)$ leave $\widetilde{D}$ fixed, we can think of them as acting on the immersion $h=f \# g$ instead of on $g$, and we can consider the sequence of transverse immersions $h=h_{0}, h_{1}, \ldots, h_{k}: S_{1} \# S_{2} \rightarrow M$ such that $h_{i}$ is obtained from $h_{i-1}$ by the pushing disk $\left(D_{i}, B_{i}\right)$. Note that $h_{k}\left(S_{1} \# S_{2}\right)=\Sigma_{1} \# \Sigma_{2}^{*}$, where $\Sigma_{1} \# \Sigma_{2}^{*}$ is obtained by piping $\Sigma_{1}$ with $\Sigma_{2}^{*}$ exactly in the same way as $\Sigma_{1}$ is piped with $\Sigma_{2}$, and then a final transverse pushing disk $\left(D_{k+1}, B_{k+1}\right)$ transforms $h_{k}$ into $f$. Thus, we can assume that there is a finite sequence of transverse pushing disks leaving $S_{1}-\delta_{1}$ fixed that transform $h=f \# g$ into $f$.

Take a good triangulation $T$ of $M$ with respect to $f, g, g_{1}, \ldots, g_{k}, h$, $h_{1}, \ldots, h_{k}$ (see Theorem 22 and Definition 23).

The triangulation $T$ shells $f$ because $f$ is a filling immersion, so consider a $T$-growth $f^{\prime}$ of $f$ such that the pipings of $\Sigma_{1}$ with the components of $T$ do not affect $\Sigma_{2}$ nor the piping between $\Sigma_{1}$ and $\Sigma_{2}$. Because $\Sigma_{1}$ and $\Sigma_{2}$ form a mutually shellable filling pair, in particular $\Sigma_{2}$ shells $\Sigma_{1}$. By Key Lemma 3 , we can take $f^{\prime}$ which also defines a $T$-growth $h^{\prime}:=f^{\prime} \# g$ of $h=f \# g$.

Consider now the sequence of immersions $h^{\prime}=h_{0}^{\prime}, h_{1}^{\prime}, \ldots, h_{k}^{\prime}, h_{k+1}^{\prime}=f^{\prime}$ such that $h_{i}^{\prime}$ is obtained from $h_{i-1}^{\prime}$ by the pushing disk $\left(D_{i}, B_{i}\right)$ for $i=$ $1, \ldots, k+1$.

Note that by construction, each $h_{i}^{\prime}$ is a $T$-inflating of $h_{i}$. Because of the choice of $T$, for each $i=1, \ldots, k+1$ the triangulation $T$ restricted to the pushing ball $B_{i}$ collapses simplicially into $g\left(D_{i}\right)=h^{\prime}\left(D_{i}\right)$. By Key Lemma 2 this implies that $h_{i}^{\prime}$ is filling homotopic to $h_{i-1}^{\prime}$ for each $i=1, \ldots, k+1$.

To sum up, $h$ is filling homotopic to $h^{\prime}$ because $h^{\prime}$ is a T-growth of $h, h^{\prime}$ is filling homotopic to $f^{\prime}=h_{k+1}^{\prime}$ by repeated application of Key Lemma 2 to the pushing disks $\left(D_{i}, B_{i}\right)$ for $i=1, \ldots, k+1$, and $f^{\prime}$ is filling homotopic

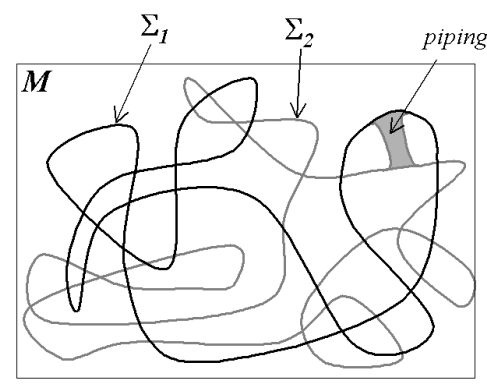

(a)

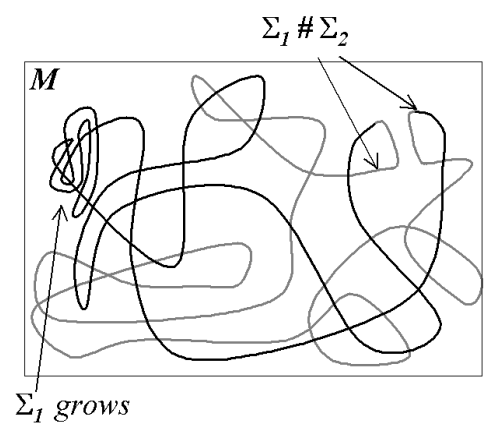

(b)

Fig. 21 


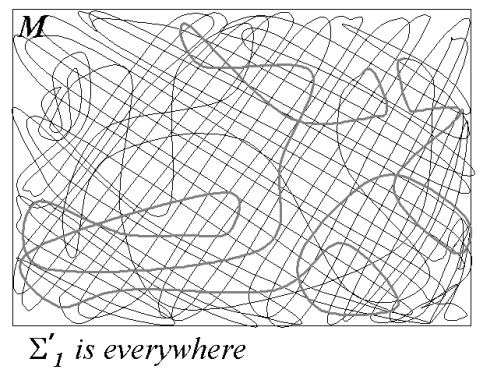

(a)

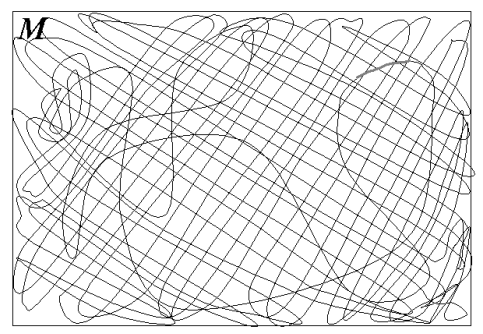

(a)

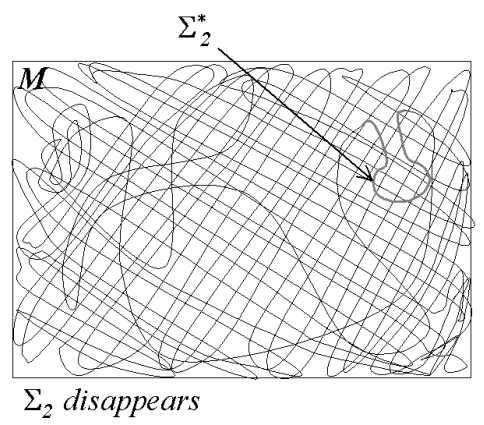

(b)

Fig. 22

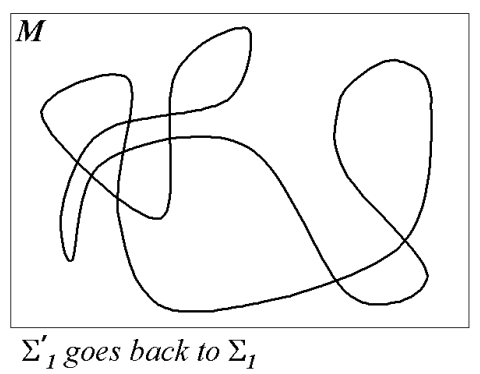

(b)

Fig. 23

to $f$ because $f^{\prime}$ is a $T$-growth of $f$. Therefore, $h$ is filling homotopic to $f$ (Figures 21-23).

By the same arguments, $h$ is filling homotopic to $g$ and thus $f$ and $g$ are filling homotopic.

12. Diagrams. Let $\Sigma \subset M$ be a Dehn surface in $M$, and let $f: S \rightarrow M$ be a parametrization of $\Sigma$. As pointed out in Section 1, the singular set $S(f)$, together with the information about how its points are identified by $f$, is what we call the Johansson diagram of $\Sigma$. We now give a more detailed definition of the Johansson diagram. This new definition is equivalent to the definition given in $[\mathrm{P}]$. We now assume for simplicity that both $S$ and $M$ are orientable.

Let $\bar{\gamma}: S^{1} \rightarrow M$ be a parametrization of a double curve of $\Sigma$. Because both $S$ and $M$ are orientable, the inverse image under $f$ of $\bar{\gamma}\left(S^{1}\right)$ is the union of two different closed curves in $S(f)$. There are exactly two different immersions $\gamma_{1}, \gamma_{2}: S^{1} \rightarrow S$ such that $f \circ \gamma_{1}=f \circ \gamma_{2}=\bar{\gamma}$. In this situation, we say that $\gamma_{1}$ and $\gamma_{2}$ are lifted curves of $\bar{\gamma}$ under $f$ and that they are sisters under $f$. 
A complete parametrization of the singularity set $\bar{S}(\Sigma)$ of $\Sigma$ is a set $\overline{\mathcal{D}}=\left\{\bar{\alpha}_{1}, \ldots, \bar{\alpha}_{m}\right\}$ of immersions from $S^{1}$ into $M$ such that: (i) each $\bar{\alpha}_{i}$ parametrizes a double curve of $\Sigma$; (ii) $\bar{\alpha}_{i}\left(S^{1}\right) \neq \bar{\alpha}_{j}\left(S^{1}\right)$ if $i \neq j$; and (iii) $\bar{S}(\Sigma)=\bigcup_{i=1}^{m} \bar{\alpha}_{i}\left(S^{1}\right)$. If $\overline{\mathcal{D}}$ is a complete parametrization of $\bar{S}(\Sigma)$ and we denote by $\mathcal{D}$ the set of all lifted curves of the curves of $\overline{\mathcal{D}}$, the map $\tau$ : $\mathcal{D} \rightarrow \mathcal{D}$ that assigns to each curve of $\mathcal{D}$ its sister curve under $f$ defines a free involution of $\mathcal{D}$. The pair $(\mathcal{D}, \tau)$ contains all the information about the singular set $S(f)$ and about how the points of $S(f)$ are identified by the map $f$ : two different points $A, B \in S$ satisfy $f(A)=f(B)$ if and only if there is a parametrized curve $\alpha \in \mathcal{D}$ and a $z \in S^{1}$ with $A=\alpha(z)$ and $B=\tau \alpha(z)$.

The pair $(\mathcal{D}, \tau)$ of the previous paragraph is the model we will presently use to define an (abstract) diagram. We have seen that every Dehn surface has an associated Johansson diagram. Thus, we can define an abstract diagram in a surface $S$ as a collection $\mathcal{D}$ of closed curves in $S$ together with a free involution $\tau: \mathcal{D} \rightarrow \mathcal{D}$ such that the curves of $\mathcal{D}$ can be coherently identified by $\tau$. Now we want to know if this diagram $(\mathcal{D}, \tau)$ is the Johansson diagram coming from a transverse immersion $f: S \rightarrow M$ of $S$ into some orientable 3-manifold $M$. If this occurs, we say that the (abstract) diagram $(\mathcal{D}, \tau)$ in the surface $S$ is realizable (cf. $[\mathrm{P}])$ and that the immersion $f$ realizes the diagram $(\mathcal{D}, \tau)$.

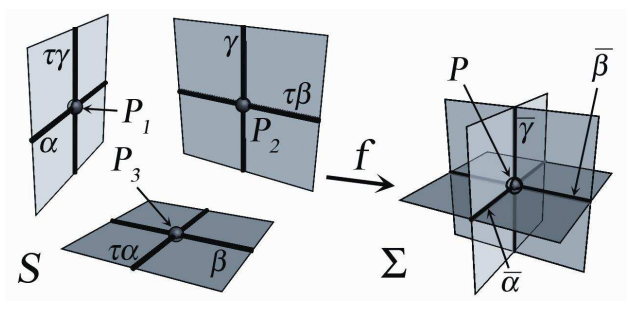

Fig. 24

The first condition that must be satisfied by the curves of $\mathcal{D}$ is that they intersect transversely as in Figure 24 at some points of $S$ which are double points of the diagram $(\mathcal{D}, \tau)$. We define two different points $A, B \in S$ to be related by the diagram $(\mathcal{D}, \tau)$ if there is a curve $\alpha \in \mathcal{D}$ and a $z \in S^{1}$ with $A=\alpha(z)$ and $B=\tau \alpha(z)$. With this terminology, each double point $A$ of the diagram will be related to two points $B, C$ of the surface $S$. If $(\mathcal{D}, \tau)$ is realizable, $B, C$ must be different and they must also be related by the diagram (see Figure 24). Thus, the double points of the diagram must be arranged in triplets of pairwise related points (the diagram is riveted in the terminology of $[\mathrm{C}]$ ). If $f: S \rightarrow M$ realizes the diagram, each of these triplets is the inverse image under $f$ of a triple point of $f$. In Figure 25 we 


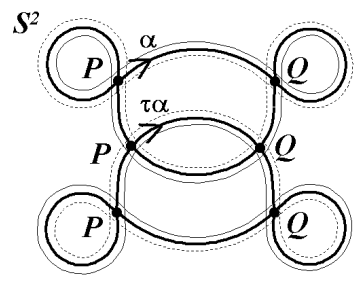

(a)

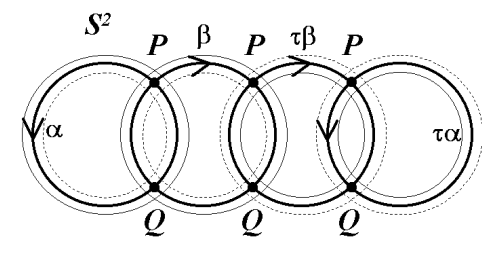

(b)

Fig. 25

have labelled the double points of each diagram in such a way that related points have the same label. We consider two diagrams on $S$ to be equivalent if they are related by a homeomorphism of $S$ or by reparametrization of the curves of the diagram.

We now recall the main result of [J2] about the realizability of diagrams. We will denote the diagram $(\mathcal{D}, \tau)$ simply by $\mathcal{D}$.

Assume that we are given an abstract diagram $\mathcal{D}$ on the surface $S$ (Figure 25). For each $\alpha \in \mathcal{D}$, we consider two neighbouring curves $\lambda, \lambda^{\prime}$ that run parallel to $\alpha$ and lie on different sides of $\alpha$ (Figure 25). We say that the two neighbouring curves of the same curve of the diagram are opposite. The neighbouring curves of the diagram can be taken such that they only intersect near the double points of the diagram and exactly as depicted in Figures 25 and 26 . We let neighbouring points of the diagram be the intersection points of the neighbouring curves with the curves of the diagram. With these assumptions, there appear four neighbouring points around each double point of the diagram (Figure 26). Consider two related double points $A, B$ of the diagram. Because they are related, there is a curve $\alpha \in \mathcal{D}$ and a $z \in S^{1}$ with $A=\alpha(z)$ and $B=\tau \alpha(z)$. If we orient the curves $\alpha, \tau \alpha$ using the standard orientation of $S^{1}$, then near $A$ the curve $\alpha$ passes through the points $A_{1}, A, A_{2}$ in this order, where $A_{1}, A_{2}$ are neighbouring points of the diagram. In the same way, near $B$ the curve $\tau \alpha$ passes through the points $B_{1}, B, B_{2}$ in this order, where $B_{1}, B_{2}$ are neighbouring points of the diagram. We assume that the neighbouring curves are so chosen that in this situation $A_{i}$ is related by the diagram to $B_{i}$ for $i=1,2$ (see Figure 26).

Once we have drawn the neighbouring curves of the diagram as in the previous paragraph, we give some definitions. If two neighbouring curves $\lambda, \mu$ pass through related neighbouring points, as the curves $\lambda$ and $\mu$ of Figure 26 , we say that $\lambda, \mu$ are elementarily related. If we orient all the curves of the diagram using the standard orientation of $S^{1}$ and if we consider the surface $S$ oriented, for a curve $\alpha \in \mathcal{D}$ we say that the neighbouring curve of $\alpha$ lying on the left-hand side of $\alpha$ is elementarily $G$-related to the neighbouring curve of $\tau \alpha$ lying on the right-hand side of $\tau \alpha$, and equivalently, that the 


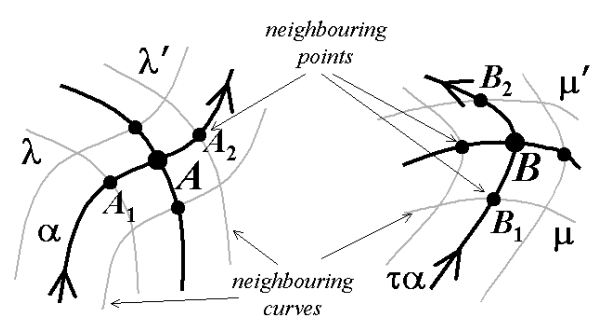

Fig. 26

neighbouring curve of $\alpha$ lying on the right-hand side of $\alpha$ is elementarily G-related to the neighbouring curve of $\tau \alpha$ lying on the left-hand side of $\tau \alpha$.

Definition 35 ([J2]). Two neighbouring curves $\lambda, \mu$ of the diagram $\mathcal{D}$ are in the same $G$-class if there exists a finite sequence $\lambda=\lambda_{0}, \lambda_{1}, \ldots, \lambda_{k}=\mu$ of neighbouring curves of the diagram such that $\lambda_{i-1}$ is elementarily related or elementarily G-related to $\lambda_{i}$ for $i=1, \ldots, k$.

In the diagrams of Figures 25(a) and 25(b) we have drawn in the same way the neighbouring curves in the same G-class. From the construction of G-classes the following can be checked without difficulty:

Lemma 36. If $\mathcal{D}$ is a diagram in $S$ and $f: S \rightarrow M$ realizes $\mathcal{D}$, then the number of $G$-classes of $\mathcal{D}$ is twice the number of connected components of the singularity set $\bar{S}(f)$.

The following theorem appears in [J2].

Theorem 37 ([J2]). A diagram $\mathcal{D}$ in the orientable surface $S$ is realizable by a transverse immersion $f: S \rightarrow M$ of $S$ into an orientable 3-manifold $M$ if and only if there are no opposite neighbouring curves of the diagram in the same G-class.

This theorem gives an easy method for checking realizability on a wide class of diagrams. An analogous result is given in $[\mathrm{C}]$ for diagrams with no closed components in surfaces with boundary.

Though Theorem 37 was stated in [J2] for diagrams in the 2-disk without singular boundary points, as pointed out in $[\mathrm{P}]$ the proof can be extended directly to the case stated here. More exactly, Theorem 37 is also true if we remove from $S$ a finite number of open disks not touching the diagram.

The key to proving Theorem 37 is 2-sidedness. Every immersion $f: S \rightarrow$ $M$ with both $S$ and $M$ orientable is 2-sided, and this 2-sidedness is reflected in the neighbouring curves of the diagram. An immersion $f: S \rightarrow M$ is 2sided if there exists an immersion $F: S \times[-1,1] \rightarrow M$ with $F(X, 0)=f(X)$ for every $X \in S$. Put $\Sigma=f(S)$. If $f$ is 2 -sided and transverse, we can choose $F$ as close to $f$ as we want, such that in a neighbourhood of a double curve 


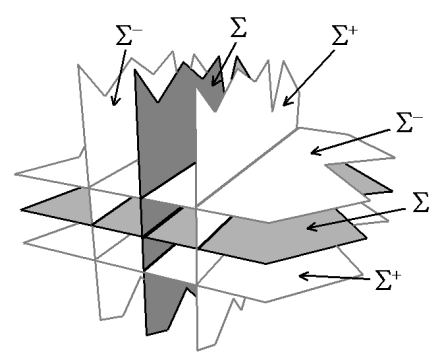

Fig. 27

of $\Sigma$ the image of $F$ looks like Figure 27. In that figure, we can see that the lower sheet $\Sigma^{-}=F(S \times\{-1\})$ and the upper sheet $\Sigma^{+}=F(S \times\{1\})$ intersect $\Sigma$ in some curves that behave exactly as the images under $f$ of the neighbouring curves of the Johansson diagram $\mathcal{D}$ of $f$. We can assume without loss of generality that in this case, the neighbouring curves of the diagram form exactly the inverse image $f^{-1}\left(\Sigma^{-} \cup \Sigma^{+}\right)$. In [J2] the following proposition is proved.

Proposition 38. If two neighbouring curves of $\mathcal{D}$ are in the same $G$ class, then their images under $f$ must be contained in the same sheet $\Sigma^{-}$ or $\Sigma^{+}$.

This implies that if $\mathcal{D}$ is realizable by a 2 -sided immersion, there cannot be two opposite neighbouring curves in the same G-class.

On the other hand, if there are not two opposite neighbouring curves of the abstract diagram $\mathcal{D}$ on $S$ in the same G-class, we can make an identification $\sim$ on the thickened surface $S \times[-1,1]$ compatible with the diagram so that neighbourhoods of sister curves are identified as in Figure 27. The quotient $\widehat{M}(\mathcal{D})=S \times[-1,1] / \sim$ is a 3 -manifold with boundary and it satisfies: (i) the canonical projection $\pi: S \times[-1,1] \rightarrow \widehat{M}(\mathcal{D})$ is an immersion; (ii) if we take the inclusion $j: S \rightarrow S \times[-1,1]$ given by $j(X)=(X, 0)$, then $\pi \circ j$ is a transverse immersion realizing $\mathcal{D}$; and (iii) $\widehat{M}(\mathcal{D})$ is orientable. See [J1] and [J2] for more details.

Going back to the immersion $f$, if it is a filling immersion, the singularity set $\bar{S}(f)$ must be connected and by Lemma 36 this implies that the Johansson diagram $\mathcal{D}$ of $f$ has only two G-classes of neighbouring curves. In this case, the manifold with boundary $\widehat{M}(\mathcal{D})$ constructed from $\mathcal{D}$ as in the previous paragraph is uniquely determined by $\mathcal{D}$ and it is homeomorphic to a regular neighbourhood of the filling Dehn surface $\Sigma \subset M$. Because $f$ is a filling immersion the boundary of $\vec{M}(\mathcal{D})$ must be a union of 2-spheres. Pasting a 3-ball to $\widehat{M}(\mathcal{D})$ along each boundary component we obtain a closed 3-manifold $M(\mathcal{D})$ homeomorphic to $M$. In this way one reconstructs a 3-manifold $M$ from a Johansson representation of $M$. 
Now assume that we are given a realizable diagram $\mathcal{D}$ in an orientable surface $S$ and that we want to know if it is the Johansson diagram of a filling Dehn sphere of some 3-manifold. First of all, the diagram $\mathcal{D}$ must fill the surface $S$ (that is, $S-\mathcal{D}$ must be a disjoint union of open 2-disks, and $\mathcal{D}-\{$ double points of $\mathcal{D}\}$ must be a disjoint union of open intervals), and in particular the curves of $\mathcal{D}$ must compose a connected graph on $S$. By Lemma 36 this implies that $\mathcal{D}$ has exactly two opposite G-classes of neighbouring curves (because $\mathcal{D}$ is realizable). As in the previous paragraph, the construction of $\widehat{M}(\mathcal{D})$ is uniquely determined by the diagram, and $\mathcal{D}$ is the Johansson diagram of a filling Dehn sphere of some 3-manifold $M$ if and only if $\partial \widehat{M}(\mathcal{D})$ is a collection of 2 -spheres. If this occurs, we say that $\mathcal{D}$ is a filling diagram, and pasting a 3-ball to $\widehat{M}(\mathcal{D})$ along each boundary component of $\widehat{M}(\mathcal{D})$ we obtain the required closed 3-manifold $M(\mathcal{D})$ that is also uniquely determined by the diagram $\mathcal{D}$. The construction of $\widehat{M}(\mathcal{D})$ can be made in an algorithmic way. The diagrams of Figures 25(a), 25(b) and 28 (a) are all examples of (realizable) filling diagrams. The diagram of Figure 25(a) appears in the original paper [J1] and it is a Johansson representation of the 3-sphere. Its corresponding filling Dehn sphere is Johansson's sphere (see Fig. 8 of [Sh]). The diagram of Figure 25(b) represents $S^{2} \times S^{1}$. The

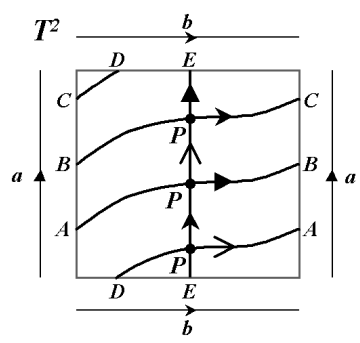

(a)

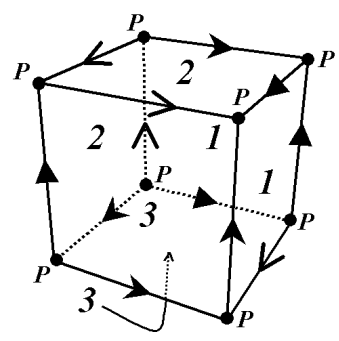

(b)

Fig. 28

diagram of Figure 28(a) is a diagram of a filling Dehn torus $\Sigma_{0}$ with only one triple point in a Euclidean 3-manifold $M$. This Euclidean manifold coincides with the Seifert manifold $M\left(S_{333}\right)=(O o 0 \mid-1 ;(3,1),(3,1),(3,1))$ (see [Mo1, p. 155]), and it is the result of identifying the faces of a solid cube in pairs as in Figure 28(b). The filling Dehn torus $\Sigma_{0}$ is the image in $M$ of the boundary of the cube under this identification.

Figure 29 shows how the Haken moves (except for finger move 0) for immersions are reflected in the Johansson diagrams. These are the diagram moves, and we label them as the corresponding moves of immersions. If we perform a diagram move in a filling diagram, the move is filling-preserving if the resulting diagram is again a filling diagram. 


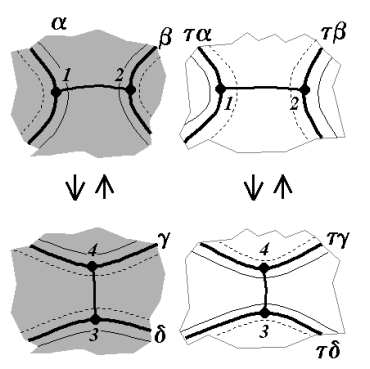

saddle move

(a)

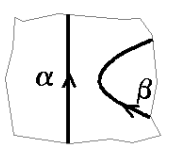

$\downarrow \uparrow$

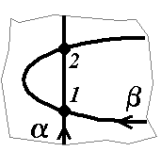

Finger move 1

(c)
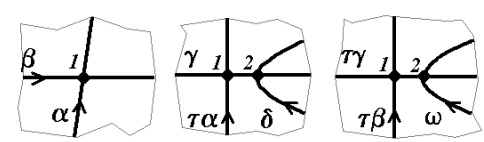

$\downarrow \uparrow$
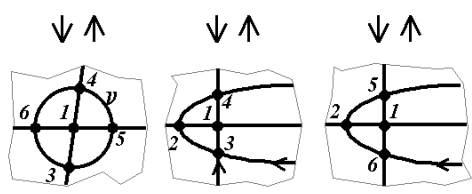

Finger move 2

(e)
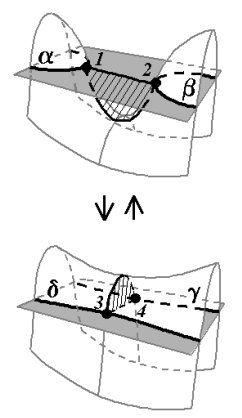

(b)

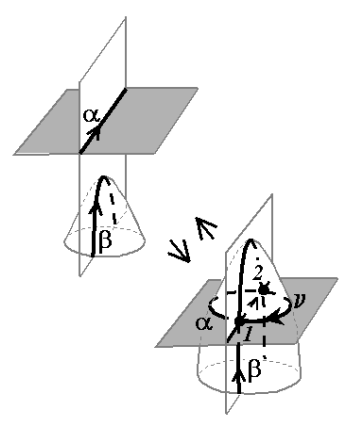

(d)

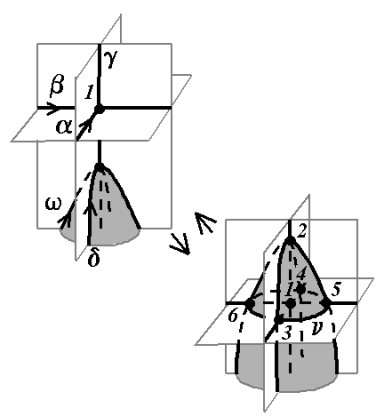

(f)

Fig. 29

Let $\mathcal{D}$ be a filling diagram on the surface $S$, and let $i: \widehat{M}(\mathcal{D}) \hookrightarrow M(\mathcal{D})$ be the inclusion map. Denote simply by $f$ the immersion $i \circ \pi \circ j: S \rightarrow M(\mathcal{D})$ that realizes $\mathcal{D}$, and put $\Sigma=f(S)$.

If we perform a filling-preserving diagram move in $\mathcal{D}$, this move will come from a filling-preserving move of $f$ and thus the new diagram $\mathcal{D}^{\prime}$ we obtain satisfies $M\left(\mathcal{D}^{\prime}\right)=M(\mathcal{D})$. As it happened with Haken moves, a finger move +1 or \pm 2 in the filling diagram $\mathcal{D}$ will always be filling-preserving, 
and a finger move -1 or a saddle move on $\mathcal{D}$ may or may not be fillingpreserving.

The neighbouring curves of the diagram help us to perform the saddle move and the finger move 1 .

A saddle move can be performed in the diagram $\mathcal{D}$ every time we have two arcs connecting related points of the diagram as in Figure 29(a). The two neighbouring curves of $\mathcal{D}$ that intersect any of the two arcs must belong to the same G-class. If we have such a pair of arcs, because $\mathcal{D}$ is a filling diagram, (the images under $f$ of) these arcs must bound a 2 -gon $w$ in $M(\mathcal{D})-$ $\Sigma$ as in Figure 29(b), and we can perform a saddle move on $f$ by pushing along $w$ any of the two sheets of $\Sigma$ that bound $w$. This saddle move on $f$ is reflected in the saddle move of the diagram $\mathcal{D}$ as depicted in Figure 29(a).

If we perform a finger move +1 on the diagram $\mathcal{D}$ there appear a new pair $\nu, \tau \nu$ of sister curves of the diagram. The diagram $\mathcal{D}$ tells us how we must identify the new double points (labelled 1 and 2 in Figure 29(c)) that appear in the new diagram $\mathcal{D}^{\prime}$, but there is some ambiguity (that does not occur for finger moves 2) because there are two ways of identifying $\nu$ with $\tau \nu$ (for a given orientation of $\nu$ there are two possible orientations of $\tau \nu$ ). This ambiguity disappears when we draw the neighbouring curves of the diagram $\mathcal{D}^{\prime}$, using the fact that related neighbouring points of the diagram must lie on neighbouring curves of the same G-class.

We say that a filling diagram $\mathcal{D}$ on a surface $S$ is nullhomotopic if the immersion $f=i \circ \pi \circ j$ as above is nullhomotopic. The diagram of Figure 25(a) is nullhomotopic (every diagram representing $S^{3}$ must be nullhomotopic), while the diagram of Figure 25(b) is not nullhomotopic.

The following result is a corollary of Theorem 2 .

COROllary 39. Two nullhomotopic filling diagrams on $S^{2}$ represent the same 3-manifold if and only if they are related by a finite sequence of filling-preserving moves.

13. Duplication. It is possible to obtain algorithmically a nullhomotopic Johansson representation of $M$ from any, nullhomotopic or not, Johansson representation $\mathcal{D}$ of $M$. We will call this process duplication of diagrams and it can be made using Johansson's construction of $\widehat{M}(\mathcal{D})$ as follows.

Let $f: S^{2} \rightarrow M$ be a filling immersion, and put $\Sigma=f(S)$. Take a thickening $F: S^{2} \times[-1,1] \rightarrow M$ of $f$ as in the previous section, such that near a double curve of $\Sigma$ the image of $F$ intersects itself as in Figure 27 , and consider the upper sheet $\Sigma^{+}=F\left(S^{2} \times\{1\}\right)$ and the lower sheet $\Sigma^{-}=F\left(S^{2} \times\{-1\}\right)$, which are two filling Dehn spheres of $M$ parallel to $\Sigma$ on both sides of $\Sigma$. The Johansson diagram $\mathcal{D}$ of $f$ has two G-classes of 
neighbouring curves. We can take the neighbouring curves of $\mathcal{D}$ such that their images under $f$ form the intersection of $\Sigma$ with $\Sigma^{-} \cup \Sigma^{+}$. We let the upper (resp. lower) G-class be the G-class of neighbouring curves of $\mathcal{D}$ whose image under $f$ is contained in the upper (resp. lower) sheet $\Sigma^{+}$(resp. $\Sigma^{-}$).

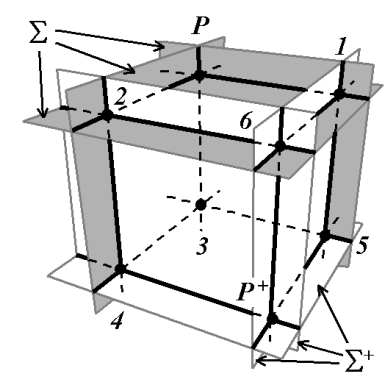

(a)

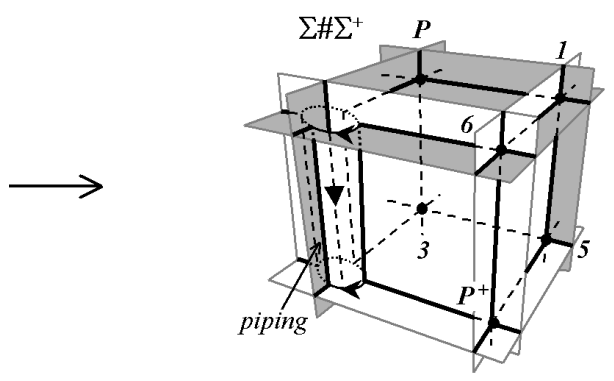

(b)

Fig. 30

The Dehn spheres $\Sigma, \Sigma^{+}$form a filling pair of spheres in $M$. Near a triple point $P$ of $\Sigma$ there will be eight triple points of the union $\Sigma \cup \Sigma^{+}$ as in Figure 30(a). In some situations the Dehn sphere $\Sigma \# \Sigma^{+}$that we obtain by piping $\Sigma$ with $\Sigma^{+}$near $P$ as in Figure 30(b) is a filling Dehn sphere of $M$. Assume that this is the case. This filling Dehn sphere $\Sigma \# \Sigma^{+}$ is the image under $F$ of the boundary of $S^{2} \times[0,1]$ with a small cylinder connecting $S^{2} \times\{0\}$ with $S^{2} \times\{1\}$ removed, and thus it is nullhomotopic.
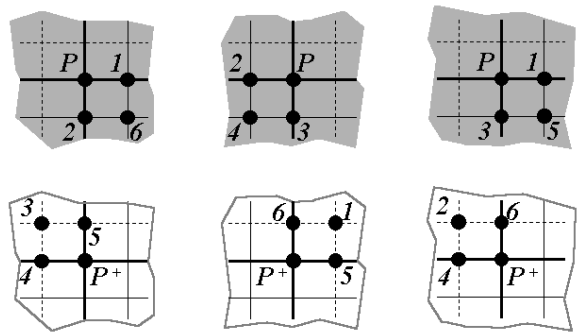

(a)
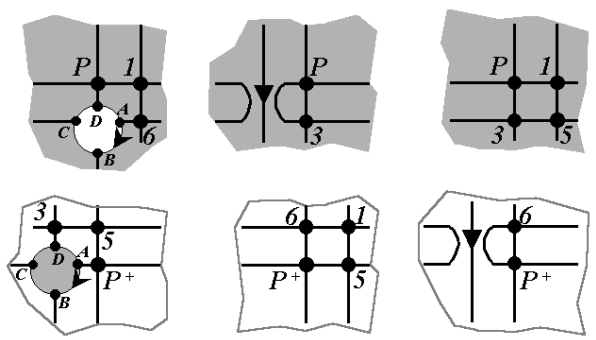

(b)

Fig. 31 
The Johansson diagram of $\Sigma \# \Sigma^{+}$can be obtained algorithmically from the Johansson diagram $\mathcal{D}$ of $\Sigma$. Let $f^{+}: S^{2} \rightarrow M$ be the parametrization of $\Sigma^{+}$given by $f^{+}(X)=F(X, 1)$.

First, remember that the upper G-class of neighbouring curves of $\mathcal{D}$ forms the inverse image under $f$ of $\Sigma \cap \Sigma^{+}$. A second observation is that the Johansson diagram of $f^{+}$is a copy $\mathcal{D}^{+}$of $\mathcal{D}$. The third observation is the following

REMARK 40. The relative position of $\Sigma$ with respect to $\Sigma^{+}$is exactly the same as that of $\Sigma^{-}$with respect to $\Sigma$.

This remark implies that if we give the G-classes of $\mathcal{D}^{+}$the same name as their respective copies in $\mathcal{D}$, the lower $G$-class of $\mathcal{D}^{+}$forms the inverse image of $\Sigma \cap \Sigma^{+}$under $f^{+}$.

The Johansson diagram $\mathcal{D} \# \mathcal{D}^{+}$of $\Sigma \# \Sigma^{+}$is what we call a duplicate of $\mathcal{D}$, and it can be obtained from $\mathcal{D}$ and $\mathcal{D}^{+}$as indicated in Figure 31 . In Figure 31(a) we depict how the cube of Figure 30(a) is seen in the Johans-
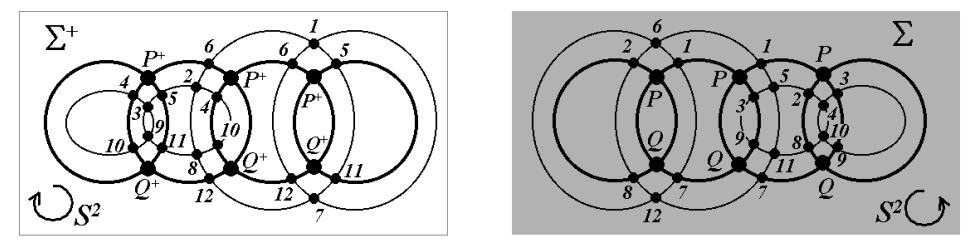

(a)
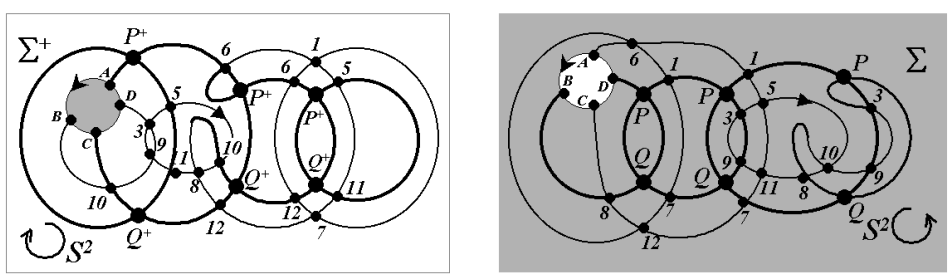

(b)

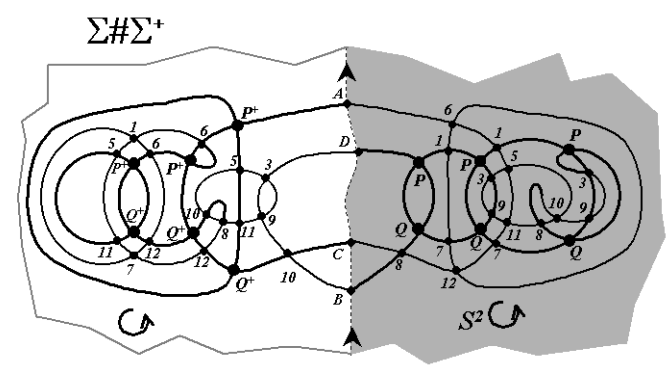

(c)

Fig. 32 
son diagrams of $\Sigma$ (grey) and $\Sigma^{+}$(white). In both diagrams we draw the lower G-class and the upper G-class similarly. As an example we apply this construction to the diagram of Figure 25(b) in Figure 32.

It is not always possible to obtain a triple point of $\Sigma$ such that if we pipe $\Sigma$ with $\Sigma^{+}$near $P$ as in Figure $30\left(\right.$ b) the resulting Dehn sphere $\Sigma \# \Sigma^{+}$ fills $M$, but we can always deform $\Sigma$ by filling-preserving moves to obtain another filling Dehn sphere $\Sigma^{\prime}$ with a triple point where the (filling-preserving) duplication is possible. This deformation can be made very easily: for example, if $P$ is one of the two triple points that appear after a finger move +1 , then duplication is possible at $P$.

Duplication allows us to give a more general version of Corollary 39:

TheOREM 41. Two filling diagrams on $S^{2}$ represent the same 3 -manifold if and only if their duplicates are related by a finite sequence of fillingpreserving moves.

\section{Miscellany}

14.1. Invariants of 3-manifolds. The first immediate application of Corollary 39 is to the search of invariants of 3-manifolds. If we could assign to each nullhomotopic diagram on $S^{2}$ an object which remains invariant under filling-preserving diagram moves, then this object defines a 3-manifold invariant. If $\varphi$ denotes such an invariant, for computing $\varphi$ for a given manifold $M$ we would need a nullhomotopic Johansson representation of $M$. If we have an arbitrary Johansson representation $\mathcal{D}$ of $M$ and we do not know if it is nullhomotopic, duplicating $\mathcal{D}$ we will be able to compute $\varphi$ from $\mathcal{D}$. However, duplication produces very complicated diagrams (the number of triple points of a duplication of $\mathcal{D}$ is eight times the number of triple points of $\mathcal{D}$ minus 2), and for this reason it should be interesting to know how to decide if a given filling diagram in $S^{2}$ is nullhomotopic or not. This is an open problem. In [Mo2] an algorithm is indicated to obtain a nullhomotopic Johansson representation of $M$ from any Heegaard diagram of $M$. A simpler algorithm is studied in detail in [V1].

14.2. The diagram group. Let $\mathcal{D}$ be a realizable diagram on $S^{2}$, and let $f: S^{2} \rightarrow M$ be a transverse immersion parametrizing $\mathcal{D}$. There is an easy way to obtain a presentation of the fundamental group of $\Sigma:=f\left(S^{2}\right)$ in terms of the diagram $\mathcal{D}$. If $\mathcal{D}=\left\{\alpha_{1}, \ldots, \alpha_{n}\right\}$, then we define the diagram group

$$
\pi(\mathcal{D})=\left|\alpha_{1}, \ldots, \alpha_{n}: \alpha_{1} \cdot \tau \alpha_{1}=\cdots=\alpha_{n} \cdot \tau \alpha_{n}=r_{1}=\cdots=r_{k}=1\right|,
$$

where the relators $r_{1}, \ldots, r_{k}$ are given by the triplets of pairwise related double points of $\mathcal{D}$. If $P_{1}, \ldots, P_{k}$ are the triple points of $\Sigma$, and $P_{j}$ is the triple point $P$ of Figure 24, which is reflected in the triplet of $\mathcal{D}$ of the same 
Figure 24, then the associated relator is

$$
r_{j}=\alpha \beta \gamma
$$

It can be proved that $\pi_{1}(\Sigma)$ is isomorphic to the diagram group [V2] and thus, if $\Sigma$ fills $M$ the diagram group gives a presentation of the fundamental group of $M$. This presentation is due to W. Haken (see Problem 3.98 of $[\mathrm{K}]$ ), and we have not seen it in printed form.

With this presentation, the following (unpublished) theorem of W. Haken can be proved (see also [Ha]). If $\mathcal{D}$ is a connected realizable diagram in $S^{2}$ with only two double curves $\alpha, \tau \alpha$, then both are simple or both are nonsimple.

THEOREM 42. If $\alpha$ and $\tau \alpha$ are simple, then $\pi(\mathcal{D}) \simeq \mathbb{Z}_{3}$, and if both are nonsimple, then $\pi(\mathcal{D}) \simeq 1$.

14.3. Checking fillingness. As we have said, to test if a realizable diagram $\mathcal{D}$ is a filling diagram is to check if $\partial \widehat{M}(\mathcal{D})$ is a collection of 2-spheres. Though the complete construction of the manifold with boundary $\widehat{M}(\mathcal{D})$ from the diagram $\mathcal{D}$ can be made in an algorithmic way using Johansson's construction, it is interesting to have faster methods for checking fillingness. The following result will give us a method for saving time in this process. It can be proved easily using Euler characteristic techniques.

LEMMA 43. A realizable diagram $\mathcal{D}$ on the genus $g$ surface $S$ is a filling diagram if and only if it fills $S$ and $\partial \widehat{M}(\mathcal{D})$ has $p+\chi(S)$ connected components, where $p$ is the number of triplets (of pairwise related double points) of $\mathcal{D}$.

The diagram group can help us also in checking fillingness. In Lemma 4.9 of [He], it is proved that if a 3-manifold with boundary $\widehat{M}$ has a boundary component which is not a 2 -sphere, then $\widehat{M}$ has a double cover. Thus,

LEMMA 44. If $\mathcal{D}$ is a realizable connected diagram in $S^{2}$ and the diagram group $\pi(\mathcal{D})$ has no subgroup of index 2 , then $\mathcal{D}$ is a filling diagram.

This lemma together with Theorem 42 gives the following.

COROLlary 45. If $\mathcal{D}$ is a realizable connected diagram in $S^{2}$ with only two curves, then it is a filling diagram.

14.4. Filling eversion. Theorem 2 applies not only to filling Dehn spheres but to their parametrizations. Let $f: S^{2} \rightarrow M$ be a filling immersion, and consider also the immersion $g: S^{2} \rightarrow M$ given by $g=f \circ a$, where $a$ now denotes the antipodal map of $S^{2}$. In this situation, the fact that $f\left(S^{2}\right)$ is filling homotopic to $g\left(S^{2}\right)$ is trivial because they are the same Dehn sphere of $M$. Theorem 2 asserts that $f$ is filling homotopic to $g$, which is now a nontrivial fact. This is a filling version of the problem of eversion of the 
2-sphere (see $[\mathrm{M}-\mathrm{P}]$ ). In [M-B] it is proved that every eversion of the 2 -sphere in $S^{3}$ has at least one quadruple point (compare also [No]). Using this, it can be seen that any parametrization $f$ of the Johansson sphere and its antipodal parametrization $g$ cannot be turned into each other by using only finger moves 1 and filling-preserving saddle moves. This means that finger move 2 cannot be dispensed with in the statement of Theorem 2 .

14.5. The nonorientable case. In our discussion about diagrams in Section 12 we have assumed that surfaces and 3-manifolds are orientable. If $f: S \rightarrow M$ is a transverse immersion and $S$ or $M$ (or both) are nonorientable, then the inverse image under $f$ of a double curve $\bar{\alpha}$ of $f$ may be a unique closed curve $\alpha$ in $S$ such that $\alpha$ is a 2-fold covering of $\bar{\alpha}$ (see [J1]). For this reason, in this general case we need a more general definition of abstract diagram. The one given in Section 12 can be adapted to this general case by allowing a nonfree involution $\tau$ and requiring that the curves $\alpha$ of the diagram with $\tau \alpha=\alpha$ commute with the antipodal map of $S^{1}$. Another fact is that in the general case, the immersion $f$ might be 1-sided, and thus the proof of Theorem 37 breaks down.

Johansson proves in [J1] a theorem characterizing diagrams in $S^{2}$ which are realizable in 3-manifolds (orientable or not). It is an interesting problem to generalize this last theorem of Johansson to cover diagrams in any surface and immersions in general 3-manifolds (orientable or not). This is certainly not very difficult but it is an intermediate step to generalize our theory of filling immersions to cover general Dehn surfaces (orientable or not) immersed in general 3-manifolds (orientable or not). This is an open program.

14.6. A question of R. Fenn. The following question was asked to us by R. Fenn (see also $[\mathrm{F}-\mathrm{R}]$ ):

Do filling Dehn surfaces in $M$ lift to embeddings in $M \times[0,1]$ ?

We do not know the complete answer to this question.

In [Gi] an algorithm is given for deciding if a Dehn surface $\Sigma$ in $\mathbb{R}^{3}$ lifts to an embedding in $\mathbb{R}^{4}$ in terms of the Johansson diagram of $\Sigma$. In the same paper there is an example of a Dehn sphere $\Sigma_{1}$ (that we will call Giller's sphere) in $\mathbb{R}^{3}$ that does not lift to an embedding in $\mathbb{R}^{4}$. The Johansson diagram of $\Sigma_{1}$ has only two nonsimple curves, and by Corollary $45, \Sigma_{1}$ will be a filling Dehn sphere of $S^{3}$. On the other hand, Johansson's example of Figure 25(a) represents a liftable (to an embedding in $\mathbb{R}^{4}$ ) filling Dehn sphere of $S^{3}$. Thus in $S^{3}$ there are liftable and nonliftable filling Dehn spheres.

A cleaner version of Giller's algorithm is Theorem 3.2 of [C-S]. This theorem can be easily adapted to the general case (see [V2]), giving a theorem that determines when a Dehn surface in $M$ lifts to an embedding in 
$M \times[0,1]$. If we apply this result to the filling Dehn spheres that arise from the algorithm given in [V1], it can be seen that every 3-manifold $M$ has a nullhomotopic filling Dehn sphere that lifts to an embedding in $M \times[0,1]$.

It remains to investigate if every 3 -manifold has a nonliftable filling Dehn sphere. Giller's sphere $\Sigma_{1}$ is a Dehn sphere in $\mathbb{R}^{3}$. It is clear that we can choose a closed 3-ball $B_{1}$ in $\mathbb{R}^{3}$ such that $\Sigma_{1} \subset B_{1}$. If $M$ is an arbitrary 3-manifold, taking an embedding from $B_{1}$ into $M$ we will obtain a copy $\Sigma_{1}^{\prime}$ of $\Sigma_{1}$ living inside $M$. By Giller's algorithm, "liftability" depends on the Johansson diagram, and the Johansson diagrams of $\Sigma_{1}^{\prime}$ and $\Sigma_{1}$ are identical. Thus, $\Sigma_{1}^{\prime}$ is a nonliftable Dehn sphere in $M$, but $\Sigma_{1}^{\prime}$ does not fill $M$ unless $M=S^{3}$. When $M$ is not $S^{3}$, we can perform a piping connecting $\Sigma_{1}^{\prime}$ with a nullhomotopic filling Dehn sphere of $M$. We conjecture that this can be done in such a way that the resulting Dehn sphere is a nonliftable nullhomotopic filling Dehn sphere of $M$.

14.7. The triple point spectrum. The minimal number of triple points of filling Dehn surfaces of a 3-manifold $M$ satisfying some particular property can be in some cases a topological invariant of $M$. We define the triple point number $t(M)$ of a closed orientable 3-manifold $M$ as the minimal number of triple points of all its filling Dehn surfaces, and the genus $g$ triple point number $t_{g}(M)$ of $M$ as the minimal number of triple points of all its genus $g$ filling Dehn surfaces. The ordered collection $\left(t_{0}(M), t_{1}(M), t_{2}(M), \ldots\right)$ of all the genus $g$ triple point numbers of the 3 -manifold $M$ for all $g \geq 0$ is what we call the triple point spectrum $\mathfrak{T}(M)$ of $M$. We can make similar definitions imposing topological restrictions on the filling Dehn surfaces considered. For example, we can define the nullhomotopic triple point number of $M$ as the minimal number of triple points of all its nullhomotopic filling Dehn surfaces; in a similar way the nullhomotopic genus $g$ triple point number or the nullhomotopic triple point spectrum can be defined. All of them are topological invariants of the 3-manifold and give a measure of the complexity of the manifold in the same way as the Heegaard genus, for example. If we have a filling Dehn surface $\Sigma$ in a 3-manifold, using pipings as in Figure 19(b), we can perhaps reduce the number of triple points of $\Sigma$, but increasing the genus of the filling Dehn surface. So there is some relation between the different genus $g$ triple point numbers that would be interesting to clarify.

Any Dehn sphere in a closed orientable 3-manifold has an even number of triple points ([Ha, p. 105]). This is not the case for genus $g>0$ Dehn surfaces, as can be seen in the example given by Figure 28(a). This means that if we want a set of moves to relate all Dehn surfaces (of any genus) of any 3-manifold, the Homma-Nagase moves introduced here, together with pipings, do not suffice because all of them are operations that preserve the parity of the number of triple points. 
We say that a genus $g$ filling Dehn surface $\Sigma$ of a 3-manifold $M$ is minimal if there is no other genus $g$ filling Dehn surface of $M$ with fewer triple points than $\Sigma$ has. Minimal filling Dehn surfaces, in particular minimal filling Dehn spheres, should have interesting properties, and their classification is another interesting problem. The classification of minimal Dehn spheres has been solved for $S^{3}$ in [Sh]. In that work, A. Shima gives in a different context six examples of Dehn spheres in $S^{3}$ with only two triple points. Three of these six examples fill $S^{3}$ (one of them is Johansson's sphere of Figure 25(a)) and they are minimal because, as we have said, any filling Dehn sphere must have at least two triple points. It can be deduced from the main theorem of [Sh] that these three examples are the unique possible minimal filling Dehn spheres in $S^{3}$.

Finally, we want to introduce a last definition. We say that a filling Dehn surface $\Sigma$ in a 3-manifold $M$ is irreducible if the only allowable fillingpreserving moves on $\Sigma$ are finger moves +1 or +2 . That is, $\Sigma$ is irreducible if any Dehn surface $\Sigma^{\prime}$ which can be obtained by performing a fillingpreserving move on $\Sigma$ has more triple points than $\Sigma$. Johansson's sphere is not irreducible, while Example 1.3 of [Sh] is irreducible. This means that minimality does not imply irreducibility. We are also interested in the converse question: are there examples of nonminimal irreducible filling Dehn surfaces?

\section{References}

[Ba] T. F. Banchoff, Triple points and surgery of immersed surfaces, Proc. Amer. Math. Soc. 46 (1974), 407-413.

[Bi] R. H. Bing, Some aspects of the topology of 3-manifolds related to the Poincaré conjecture, in: Lectures on Modern Mathematics, Vol. II, Wiley, New York, 1964, 93-128.

[B-M] H. Bruggesser and P. Mani, Shellable decompositions of cells and spheres, Math. Scand. 29 (1971), 197-205.

[C] J. S. Carter, Extending immersions of curves to properly immersed surfaces, Topology Appl. 40 (1991), 287-306.

[C-S] J. S. Carter and M. Saito, Surfaces in 3-space that do not lift to embeddings in 4-space, in: Knot Theory (Warsaw, 1995), Banach Center Publ. 42, Inst. Math., Polish Acad. Sci., Warsaw, 1998, 29-47.

[Ch] D. R. J. Chillingworth, Collapsing three-dimensional convex polyhedra, Proc. Cambridge Philos. Soc. 63 (1967), 353-357.

[F-R] R. Fenn and C. Rourke, Nice spines on 3-manifolds, in: Topology of Low-Dimensional Manifolds, Lecture Notes in Math. 722, Springer, 1977, 31-36.

[Gi] C. A. Giller, Towards a classical knot theory for surfaces in $\mathbb{R}^{4}$, Illinois J. Math. 26 (1982), 591-631.

[Gl] L. C. Glaser, Geometrical Combinatorial Topology, Vol. I, Van Nostrand Reinhold, New York, 1970. 
[G-P] V. Guillemin and A. Pollack, Differential Topology, Prentice-Hall, Englewood Cliffs, NJ, 1974.

[Ha] W. Haken, Some special presentations of homotopy 3-spheres, in: Topology Conference (Blacksburg, VA, 1973), Lecture Notes in Math. 375, Springer, 1974, $97-107$.

[H-H] J. Hass and J. Hughes, Immersions of surfaces in 3-manifolds, Topology 24 (1985), 97-112.

[He] J. Hempel, 3-Manifolds, Ann. of Math. Stud. 86, Princeton Univ. Press, Princeton, NJ, and Univ. of Tokyo Press, Tokyo, 1976 (3rd printing, 1988).

[Hi] M. W. Hirsch, Differential Topology, Grad. Texts in Math. 33, Springer, New York, 1976.

[H-N1] T. Homma and T. Nagase, On elementary deformations of maps of surfaces into 3-manifolds, Yokohama Math. J. 33 (1985), 103-119.

[H-N2] T. Homma and T. Nagase, On elementary deformations of maps of surfaces into 3-manifolds, II, in: Topology and Computer Science, Kinokuniya, Tokyo, 1987, $1-20$.

[Hu] J. F. P. Hudson, Piecewise Linear Topology, Univ. of Chicago Lecture Notes, Benjamin, New York, 1969.

[H-Z] J. F. P. Hudson and E. C. Zeeman, On combinatorial isotopy, Inst. Hautes Études Sci. Publ. Math. 19 (1964), 69-94.

[J1] I. Johansson, Über singuläre Elementarflächen und das Dehnsche Lemma, Math. Ann. 110 (1935), 312-320.

[J2] - Über singuläre Elementarflächen und das Dehnsche Lemma II, ibid. 115 (1938), 658-669.

[K] R. Kirby, Problems in low-dimensional topology, available at http://math.berkeley.edu/ kirby/

[L] B. H. Li, On immersions of manifolds in manifolds, Sci. Sinica Ser. A 25 (1982), 255-263.

[Ma] W. S. Massey, A Basic Course in Algebraic Topology, Grad. Texts in Math. 127, Springer, New York, 1991.

[M-B] N. Max and T. Banchoff, Every sphere eversion has a quadruple point, in: Contributions to Analysis and Geometry (Baltimore, 1980), Johns Hopkins Univ. Press, Baltimore, 1981, 191-209.

[Mo1] J. M. Montesinos, Classical Tessellations and Three-Manifolds, Universitext, Springer, Berlin, 1987.

[Mo2] J. M. Montesinos-Amilibia, Representing 3-manifolds by Dehn spheres, in: Contribuciones Matemáticas: Homenaje a Joaquín Arregui Fernández, Editorial Complutense, 2000, 239-247.

[M-P] B. Morin et J.-P. Petit, Problématique du retournement de la sphère, C. R. Acad. Sci. Paris Sér. A-B 287 (1978), A767-A770.

[Mu J. R. Munkres, Elementary Differential Topology, Ann. of Math. Stud. 54, Princeton Univ. Press, Princeton, NJ, 1966.

[No] T. Nowik, Quadruple points of regular homotopies of surfaces in 3-manifolds, Topology 39 (2000), 1069-1088.

[P] C. D. Papakyriakopoulos, On Dehn's Lemma and the asphericity of knots, Ann. of Math. (2) 66 (1957), 1-26.

[R] D. Roseman, Reidemeister-type moves for surfaces in four-dimensional space, in: Knot Theory (Warsaw, 1995), Banach Center Publ. 42, Inst. Math., Polish Acad. Sci., Warsaw, 1998, 347-380. 
[R-S] C. P. Rourke and B. J. Sanderson, Introduction to Piecewise-Linear Topology, reprinted, Springer, Berlin, 1982.

[Ru] M. E. Rudin, An unshellable triangulation of a tetrahedron, Bull. Amer. Math. Soc. 64 (1958), 90-91.

[S] D. E. Sanderson, Isotopy in 3-manifolds. I. Isotopic deformations of 2-cells and 3-cells, Proc. Amer. Math. Soc. 8 (1957), 912-922.

[Sh] A. Shima, Immersed 2-spheres in the 3-sphere with only two triple points, J. Knot Theory Ramif., to appear.

[Ve] A. Verona, Stratified Mappings-Structure and Triangulability, Lecture Notes in Math. 1102, Springer, 1984.

[V1] R. Vigara, A new proof of a theorem of J. M. Montesinos, J. Math. Sci. Univ. Tokyo 11 (2004), 325-351.

[V2] - Representación de 3-variedades por esferas de Dehn rellenantes, $\mathrm{PhD}$ Thesis, UNED, Madrid, 2006.

[Wh1] J. H. C. Whitehead, Simplicial spaces, nuclei and m-groups, Proc. London Math. Soc. 45 (1939), 243-327.

[Wh2] -, On $C^{1}$-complexes, Ann. of Math. (2) 41 (1940), 809-824.

Departamento de Matemáticas Fundamentales

Facultad de Ciencias

U.N.E.D.

C/ Senda del rey 9

28040 Madrid, Spain

E-mail: rvigara@mat.uned.es

Received 3 June 2004;

in revised form 3 February 2005 